\title{
Numerical Simulation of a Full Helicopter Configuration Using Weak Fluid-Structure Coupling
}

\author{
Markus Dietz* Walid Khier † Björn Knutzen, $\stackrel{+}{;}$ \\ Siegfried Wagner ${ }^{\S}$ and Ewald Krämer ף \\ Institute of Aerodynamics and Gasdynamics, University of Stuttgart \\ Pfaffenwaldring 21, 70569 Stuttgart, Germany \\ Institute of Aerodynamics and Flow Technology, German Aerospace Center \\ Lilienthalplatz 7, 38108 Braunschweig, Germany \\ Eurocopter Deutschland GmbH \\ 81663 München, Germany
}

In this paper a weak coupling method for the main rotor between Computational Fluid Dynamics (CFD) and Computational Structural Dynamics (CSD) is presented and applied to the simulation of a complete helicopter configuration. The main focus of the paper is to assess the applicability of the method in combination with a complete helicopter configuration and to investigate the influence of the remaining helicopter components on the trimmed rotor solution. The aeroelastic method uses the CFD code FLOWer for the simulation of the aerodynamics and the flight mechanics code HOST for the simulation of the blade dynamics and the for rotor trim procedure. The numerical part of the paper adresses both the coupling and trim procedure, and the grid deformation process incorporated into the CFD method.

The aeroelastic method is applied to three different flight cases covering the typical helicopter flight regime: A low-speed flight characterized by the pitch-up phenomenon, a fast forward flight condition characterized by the tail-shake phenomenon, and a highly-loaded rotor case. The configuration considered is a wind tunnel model with powered $4.2 \mathrm{~m}$ four-bladed main rotor and $0.73 \mathrm{~m}$ two bladed tail rotor. For all three flight cases isolated rotor results are compared to the results obtained for the complete helicopter configuration. Whereas only minor differences between the isolated rotor computation and the complete helicopter configuration have been observed for the first two flight cases, the the highly-loaded rotor study revealed a significant increase in rotor power consumption of the complete configuration compared to the isolated rotor. The change in rotor power consumption was found to be related to the occurance of Dynamic Stall in case of the complete helicopter configuration.

\section{Notation}

Symbols

$C_{F z} M a^{2} \quad$ sectional thrust coefficient (upwards positive) [-]

$C_{F y} M a^{2} \quad$ sectional circumferential force coefficient (positive trailing to leading edge) [-]

$C_{M x} M a^{2} \quad$ sectional pitching moment coefficient (positive nose up) [-]

$C_{m} M a^{2}$

$C_{n} M a^{2}$

$F_{x} \quad$ rotor longitudinal force [N] (positive to aft)

$F_{y} \quad$ rotor lateral force $[\mathrm{N}]$ (positive to starboard)

$F_{z} \quad$ rotor vertical force [N] (upward positive)

\footnotetext{
*Former Research Assistant, Institute of Aerodynamics and Gasdynamics, now Eurocopter Deutschland GmbH

${ }^{\dagger}$ Researcher, Institute of Aerodynamics and Flow Technology

${ }^{\ddagger}$ Eurocopter Deutschland GmbH

$\S$ Former Head of Institute, Institute of Aerodynamics and Gasdynamics

ฯ Head of Institute, Institute of Aerodynamics and Gasdynamics
} 


\begin{tabular}{|c|c|}
\hline$M a_{\infty}$ & free stream Mach number [-] \\
\hline$M a_{\text {Tip }}$ & hover tip Mach number [-] \\
\hline$\alpha_{f}$ & fuselage pitch attitude $\left[^{\circ}\right]$ \\
\hline$\alpha_{q}$ & rotor shaft pitch attitude $\left[^{\circ}\right]$ \\
\hline$\beta_{0}$ & constant flap angle in flap hinge $\left[^{\circ}\right]$ (upwards negative) \\
\hline$\beta_{C}$ & longitudinal flap angle in flap hinge $\left[^{\circ}\right]$ (upwards negative) \\
\hline$\beta_{S}$ & lateral flap angle in flap hinge $\left[^{\circ}\right]$ (upwards negative) \\
\hline$\delta_{0}$ & constant lag angle in flap hinge $\left[^{\circ}\right]$ (forward positive) \\
\hline$\delta_{C}$ & longitudinal lag angle in flap hinge $\left[^{\circ}\right]$ (forward positive) \\
\hline$\delta_{S}$ & lateral flap lag in flap hinge $\left[^{\circ}\right]$ (forward positive) \\
\hline$\mu$ & advance ratio $[-]$ \\
\hline$\theta_{0}$ & collective pitch angle $\left[^{\circ}\right]$ \\
\hline$\theta_{C}$ & lateral cyclic pitch angle $\left[^{\circ}\right]$ \\
\hline$\theta_{S}$ & longitudinal cyclic pitch angle $\left[^{\circ}\right]$ \\
\hline$\psi$ & rotor azimuth angle $\left[^{\circ}\right]$ \\
\hline
\end{tabular}

\section{Acronyms}

$\begin{array}{ll}\text { CFD } & \text { Computational Fluid Dynamics } \\ \text { CSD } & \text { Computational Structural Dynamics } \\ \text { DOF } & \text { Degree Of Freedom } \\ \text { GOAHEAD } & \text { Generation of Advanced Helicopter Experimental Aerodynamic Database } \\ \text { HLDS } & \text { Highly loaded rotor in Dynamic Stall } \\ \text { HOST } & \text { Helicopter Overall Simulation Tool } \\ \text { HSTS } & \text { Hig-Speed Tail-Shake } \\ \text { LSPU } & \text { Low-Speed Pitch-Up }\end{array}$

\section{Introduction}

Within the past years helicopter main rotor aerodynamics and aeroelasticity has been an extensive field of research at the Institut für Aerodynamik und Gasdynamik (IAG) and the Institut für Aerodynamik und Strömungstechnik (DLR-AS). Although even the plain aerodynamic simulation of a helicopter main rotor in forward flight remains a challanging task for CFD, it has become evident that aeroelastic effects have to be taken into account in order to even get quantitatively correct results.

Different concepts can be applied in order to couple aerodynamics and structural dynamics. The coupling may either be performed on a time-step basis (denoted as strong coupling) or by making use of the periodicity of the problem (weak coupling). At the IAG extensive research on the strong coupling approach has been performed during the past years [1-3]. In case of strong coupling the data exchange between fluid and structure is carried out in a time-accurate fashion, i.e. the transfer of fluid loads from CFD to the structure code and the transfer of the blade deformation from the structure code to CFD is performed within every time step. The advantage of the strong coupling method is that the real physics of the coupling process is reproduced. Therefore the method can be applied to any flight condition including non-periodic flight cases like manoeuvering flight. On the other hand this is also a disadvantage for flight conditions where periodicity of the solution can be implied. The method reproduces the complete transient phenomenon which is mostly not of interest. This transient process can take up to eight to ten rotor revolutions until a periodic state has been obtained. Furthermore, the trim of a strongly coupled aeroelastic simulation is a very time-consuming task [3].

A method allowing for a faster convergence in case of a periodic flight condition is the so called weak (or loose) coupling. This coupling method is nowadays widely spread and has been applied to isolated rotors by several researchers $[1,4,8-11,24,27]$. The method is characterized by the exchange of periodic solutions between CFD and CSD/flight mechanics in either directions. Furthermore the trim of the rotor towards a prescribed objective is a direct outcome of the method.

The rotor trim is the prerequisite in order to allow for a meaningful comparison of the numerical results to experimental or flight test data. Both the aerodynamic and the dynamic characteristics of the rotor will only be correctly captured if the global rotor loads correspond to the ones present in the experiment. It is therefore not sufficient to prescribe the experimental rotor control angles for the coupled numerical simulation. Due to various deficiencies in the numerical modeling (simplifications of the geometry of the configuration, deficiencies in turbulence modeling and wake conservation, etc.) the 
global time-averaged rotor loads will not match the experimental ones, leading to a different overall rotor characteristic. The need for the rotor trim of a complete helicopter configuration has been demonstrated for the HELINOVI configuration where rigid blade assumption without rotor trim lead to considerable deviations from the experimental results [18].

The weak coupling method has been previously applied to isolated rotors both at DLR-AS and IAG. At DLR-AS the FLOWer code was coupled to the comprehensive code S4 [24]. At the IAG the weak coupling procedure between FLOWer and HOST has been established and applied to several test cases including active rotors [8-11]. Within the last two years the weak coupling methodology between FLOWer and HOST has been introduced into an industrial invironment at Eurocopter Deutschland $\mathrm{GmbH}$ and successfully applied to performance investigations on passive and active rotors [4].

As the next step towards a further improvement of the computational model we have recently started to extend our investigations from isolated rotor computations towards the simulation of full helicopter configurations including fuselage, rotor head and tail rotor. At the current stage of development the consideration of these additional components is restricted to the aerodynamic modelling by adding additional components to the CFD grid system. Fluid-structure coupling and trim is performed for the main rotor, i.e. the aerodynamic loads acting on the additional components are not taken into account for the coupling and trim process. This trim strategy is in line to the usual experimental trim procedure prescribing a trim state only for the main rotor of the experimental configuration.

The investigations presented in the present paper are embedded into the so-called "Blind-Test" phase of the EU project GOAHEAD. In the frame of this project a complete helicopter configuration is going to be experimentally investigated in the Large Low Speed Facility of the DNW wind tunnel. The project is intended to generate a comprehensive experimental database especially conceived to validate CFD codes for helicopter related applications. An overview on the GOAHEAD project is given in [25] and [6]. Both IAG, DLR and Eurocopter Deutschland are project partners and contribute to the CFD work package of the project. A first paper of the authors comparing the flow around the isolated fuselage to the flow around the complete GOAHEAD configuration was published in [19].

The present paper adresses three flight cases of the GOAHEAD project: A low-speed flight characterized by the pitch-up phenomenon (LSPU), a fast forward flight condition characterized by the tail-shake phenomenon (HSTS), and a highly-loaded rotor case (HLDS). The LSPU test case has been computed at IAG, the HSTS test case and the complete helicopter in HLDS condition have been computed at DLR, and the isolated rotor computations in HLDS condition have been carried out at Eurocopter Deutschland. All partners of this paper used the weak coupling methodology between FLOWer and HOST for their aeroelastic simulations. The main focus of the paper is to assess the applicability of the method in combination with a complete helicopter configuration and to investigate the influence of the remaining helicopter components on the trimmed rotor solution.

\section{Numerical Methods}

\section{A. Computational Structure Dynamics Model}

The CSD and trim part of the coupled aeroelastic analysis is carried out by the Eurocopter flight mechanics code HOST (Helicopter Overall Simulation Tool) [5]. HOST enables both the simulation of isolated helicopter components and the simulation of the full helicopter. As a general purpose flight mechanics tool HOST is able to trim the rotor based on a comparatively simple internal aerodynamics model based on lifting line theory and 2D airfoil tables. Optionally several semi-empirical corrections are available in order to improve the simulation of the aerodynamics. The distribution of the induced velocity on the rotor disc can either be modelled using an analytic approach (e.g. Meijer-Drees) or HOST may be coupled to both prescribed or free wake models. For the purpose of coupling to CFD the choice of the HOST-internal aerodynamic modeling parameters is of less relevance as the HOST-internal aerodynamics will be replaced by CFD aerodynamics during the weak coupling process.

The elastic blade model in HOST considers the blade as a quasi one-dimensional Euler-Bernoulli beam. It allows for deflections in flap and lag direction and elastic torsion along the blade axis. In addition to the assumption of a linear material law, tension elongation and shear deformation are neglected. However, possible offsets between the local cross-sectional centre of gravity, tension centre and shear centre are accounted for, thus coupling bending and torsional DOFs. The blade model is based on a geometrically non-linear formulation, connecting rigid segments through virtual joints. At each joint, elastic rotations are permitted about the lag, flap and torsion axes. Since the use of these rotations as degrees of freedom would yield a rather large system of equations, the number of equations is reduced by a modal Rayleigh-Ritz approach. A limited set of mode-like deformation shapes together with their weighting factors are used to yield a deformation description. Therefore, any degree of freedom can be 
expressed as

$$
h(r, \psi)=\sum_{i=1}^{n} q_{i}(\psi) \cdot \hat{h}_{i}(r)
$$

where $n$ is the number of modes, $q_{i}$ the generalized coordinate of mode $i$ (a function of the azimuth angle $\psi$ ), and $\hat{h}_{i}$ is the modal shape (a function of the radial position $r$ ).

\section{B. Computational Fluid Dynamics Model}

The DLR FLOWer code is used for the simulation of the aerodynamics. FLOWer solves the threedimensional, unsteady Euler or Reynolds-averaged Navier-Stokes equations (RANS) in integral form. The equations are derived for a moving frame of reference. Turbulence can be modelled either by algebraic or by transport equation models.

FLOWer uses a finite volume formulation on block-structured meshes. Both central and several upwind schemes are available for the spatial discretization. For the present investigations the central scheme was applied using a cell-centered formulation. Following the approach of Jameson [14] dissipative fluxes are explicitly added in order to damp high frequency oscillations. On smooth meshes, the scheme is formally of second order in space.

The time integration is performed by a multi-step Runge-Kutta method. Several acceleration techniques, such as implicit residual smoothing, local time-stepping and multigrid may be applied to speed up the convergence. For unsteady problems the Dual-Time-Stepping approach of Jameson [15] is used which transfers the implicit integration in physical time into the solution of a steady-state problem in an artificial time. The modified residual in artificial time is driven towards zero using Runge-Kutta integration in the same fashion as for steady-state problems. Thus all convergence acceleration methods given above remain applicable.

FLOWer is capable of calculating flows on moving grids (arbitrary translatory and rotatory motion). For this purpose the RANS equations are transformed into a body-fixed rotating and translating frame of reference. The Arbitrary-Lagrangian-Eulerian (ALE) method in conjuction with the Geometric Conservation Law (GCL) allows for the treatment of flexible meshes.

FLOWer allows for the definition of an arbitrary number of individual block structures, each of which may consist of several blocks and underlie an individual motion law. The data exchange between the blocks of a multiblock structure is peformed via ghost layers. The data exchange between the individual multiblock structures is made possible by the Chimera technique of overlapping grids $[23,28,29]$. FLOWer uses two ghost layers and two Chimera fringe layers covering the full stencil of the convective and dissipative flux computation. The Chimera connectivity is established by interpolation on block or hole boundary points. The code features full Chimera capability: Each block structure may provide donor cells to every other block structure. The search for donor cells on non-cartesian meshes is performed by the ADT method. Further information on the code can be found in [22] and [26].

\section{Grid Deformation Process}

During runtime of the CFD computation the blade dynamics have to be taken into account by a grid deformation of the blade grid structures at the beginning of each physical time step. Different strategies can be applied in order to perform the deformation process: One possibility is to generate an updated grid by means of an external grid generator. This option drops out due to the high computational effort and the need to read in 3D data from an external method during runtime. Another possibility is to interpret the grid as an elastomechanic model and to solve the equilibrium problem resulting from the prescribed outer block boundary. This method is very robust and can be applied to both structured and unstructured meshes. However, the method requires the solution of a system of equations and thus the computational effort is high.

As the deformation has to be carried out at each time step performance is the most important request to the deformation tool. For this reason we use a grid deformation tool on an algebraic basis. Algebraic methods are very fast due to their low numerical complexity (as they are non-iterative). The grid deformation tool applied in the present paper has been originally developed by Hierholz $[12,13]$ as a pure monoblock deformation tool. The tool has been implemented into FLOWer by Altmikus [3] and has recently been used for weak coupling on isolated main rotors [4,8-11]. In order to allow for a more general applicability of the tool and to account for more complex blade geometries the deformation tool has been extended towards the treatment of multiblock topologies. In the following we will describe the major characteristics of the method by means of Figure 1 which shows the deformation process of a simple sample grid. 


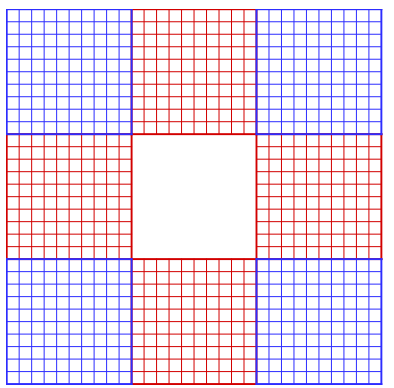

(a) Original Mesh

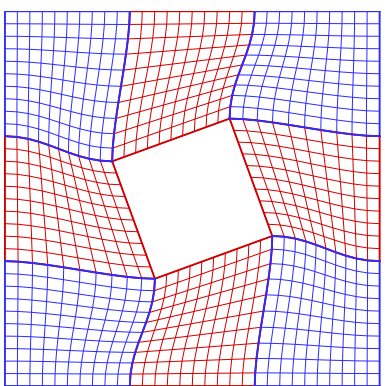

(b) Deformation using only translation

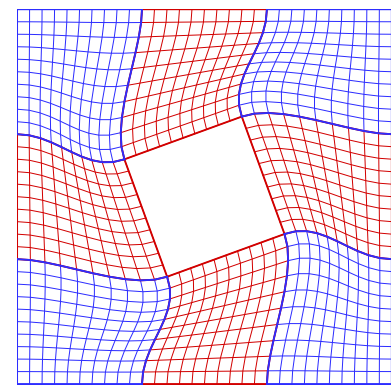

(c) Deformation using translation and rotation

Figure 1. Illustration of grid deformation process

\section{Treatment of blocks contributing to the blade surface}

The blade surface may be composed from patches belonging to several different blocks. After the reconstruction of the deformed blade surface the new location of the surface patches relative to their original location is known. This deformation is reduced towards zero along the body-normal grid lines leading from the surface to the outer block boundary. Transfinite interpolation using Hermite polynomials is applied for this process.

The method splits the deformation description into two parts: A global part associated to the complete spanwise blade section - this part is equivalent to a global translation and rotation of the rigid airfoil, and a local part associated to each grid point on the spanwise section - this part is equivalent to a shape modification of the airfoil. This allows for individual depths of penetration into the grid for either parts. Let's assume the kinematic state of a spanwise section is given by a transformation structure (reconstructed from the HOST deformation description) consisting of a shift vector $r$ and a rotation matrix $\mathbf{F}$. Hence a point of the blade surface on the spanwise section $x^{0}$ at its original location is transferred to its deformed location $x^{1}$ by

$$
\begin{gathered}
x^{1}=\mathbf{F} \cdot x^{0}+r \\
\Delta x^{01}=x^{1}-x^{0}=\mathbf{F} \cdot x^{0}+r-x^{0}
\end{gathered}
$$

If the airfoil shape is fixed this is the final location of the surface point in deformed state. If we allow for an additional shape modification of the airfoil (e.g. caused by the deflection of a flap) we have to apply an additional transformation from $x^{1}$ to the final location of the surface point $x^{2}$ :

$$
\Delta x^{12}=x^{2}-\left(\mathbf{F} \cdot x^{0}+r\right)
$$

As the final location of the surface point is known the next step is to spread the disturbance into the grid along the respective body-normal grid line. The splitting into the global and the local deformation part allows for individual penetration depths into the grid. In order to conserve the grid quality within the cells of the boundary layer (i.e. to conserve the angle of the grid lines relative to the surface normal), Hermite interpolation for the global part is applied for both translation and rotation. For the global translation we get

$$
\Delta x_{i, j, k}^{\text {trans,glob }}=\Delta x^{01} \cdot\left(1-3 g_{i, j, k}^{2}+2 g_{i, j, k}^{3}\right)
$$

assuming that $j$ is the body-normal grid index, $j=j_{\min }$ is the body surface and $g_{i, j, k}$ is the parameterization along the $j$-direction with $g=0$ at the surface and $g=1$ at the outer boundary. Hence, using the Hermite polynomial for a left-sided unit shift $1-3 g^{2}+2 g^{3}$ we get a shift of $\Delta x^{01}$ at the surface and zero shift at the outer boundary. The resulting grid deformation is illustrated by the red blocks in Figure 1(b).

In order to conserve the direction of the grid lines leaving the surface, and hence improve the grid quality of the deformed mesh, an additional shift due to rotation is superimposed: 


$$
\begin{gathered}
n_{i, j_{\min }, k}=x_{i, j_{m i n}+1, k}^{0}-x_{i, j_{m i n}, k}^{0} \\
\Delta x_{i, j, k}^{r o t, g l o b}=\frac{\mathbf{F} \cdot n_{i, j_{\min }, k}-n_{i, j_{m i n}, k}}{h_{i, j_{m i n}+1, k}} \cdot\left(h_{i, j, k}-2 h_{i, j, k}^{2}+h_{i, j, k}^{3}\right)
\end{gathered}
$$

where $h_{i, j, k}$ is the parameterization along the $j$-direction. $h-2 h^{2}+h^{3}$ is the Hermite polynomial providing a left-sided unit slope. The resulting grid deformation is given in Figure 1(c). The improvement with respect to Figure 1(b) is clearly visible.

Finally, if a shape change of the airfoil is to be considered local translation has to be taken into account:

$$
\Delta x_{i, j, k}^{\text {trans }, l o c}=\Delta x^{12} \cdot\left(1-3 g_{i, j, k}^{2}+2 g_{i, j, k}^{3}\right)
$$

The final shift of a grid point from its original location to its deformed location is thus obtained as:

$$
\Delta x_{i, j, k}=x_{i, j, k}^{\text {trans,glob }}+\Delta x_{i, j, k}^{\text {rot }, \text { glob }}+\Delta x_{i, j, k}^{\text {trans }, l o c}
$$

Up to now we have not yet defined the parameterizations $g$ ang $h$ along the body normal grid lines. The original implementation of the deformation tool was designed for Euler grids. In order to account for the grid clustering within the boundary layer of RANS meshes the parameterizations have to be adapted. For the both global and local translation a curve length parameterization along the body-normal grid lines has proven to work well:

$$
g_{i, j, k}=\frac{s_{i, j, k}}{s_{i, j_{\max }, k}}
$$

$s_{i, j, k}$ is the curve length along the body-normal grid line from the surface point $i, j_{\text {min }}, k$ to point $i, j, k$. For the global rotation, the penetration depth has to be limited in order to avoid too strong displacements of the grid points. The parameterization $h$ is obtained from $g$ as follows:

$$
h_{i, j, k}=1-\left(1-g_{i, j, k}\right)^{\alpha}
$$

A value of 128 for $\alpha$ has proven to work well for most grid configurations.

Note, that the current approach does not take into account a rotational contribution to the local deformation part. This means a shape modification of the airfoil does not influence the angle of the body-normal grid lines leaving the blade surface. As the changes in airfoil shape can generally be considered as small compared to the translation and rotation of the blade section due to fluid-structure coupling, the consideration of a local contribution to the rotational part has turned out to be unnecessary.

As stated earlier the blade surface may be composed from patches belonging to several blocks. During the deformation process it is mandatory that cuts between adjacent blocks are mapped onto each other in deformed state. As both the shift vector $r$ and the rotation matrix $\mathbf{F}$ provided from HOST are distinct functions of the radial stations the deformed state obtained for the block boundaries of adjacent blocks will be identical and hence cuts will be mapped onto each other.

\section{Treatment of blocks not contributing to the blade surface}

The basic idea of the multiblock deformation is to use the deformed blocks faces of the blocks contributing to the blade surface as a basis for the deformation of those blocks not contributing to the blade surface. Hence, in the first step the blocks contributing to the blade surface are deformed according to the procedure given above. In the second step the deformed cut faces are used as prescribed deformation for adjacent blocks not contributing to the surface. By means of this procedure it is possible to sequentially sweep over all blocks and to deform those blocks for which predeformed cut faces are available, until all blocks of the blade grid structure have been deformed.

For the deformation of the blocks not contributing to the blade surface only the translation part is taken into account for the transfinite interpolation. If several predeformed block faces on different index planes are available the deformation process is carried out sequentially along the individual index directions, starting with the $i$-direction:

$$
\Delta x_{[\mathbf{i}], i, j, k}=\Delta x_{i_{m i n}, j, k} \cdot\left(1-3 g_{[\mathbf{i}] i, j, k}^{2}+2 g_{[\mathbf{i}] i, j, k}^{3}\right)+\Delta x_{i_{m a x}, j, k} \cdot\left(3 g_{[\mathbf{i}] i, j, k}^{2}-2 g_{[\mathbf{i}] i, j, k}^{3}\right)
$$

$\Delta x_{i_{m i n}, j, k}$ denotes the displacement of a grid node $i_{m i n}, j, k$ at the $i=i_{m i n}$-face of the block and $\Delta x_{i_{\max }, j, k}$ denotes the displacement of a grid node $i_{\max }, j, k$ at the $i=i_{\max }$-face of the block. $g_{[\mathrm{i}]}$ is the 
curve lenght parameterization (computed from the undeformed grid) in $i$-direction. If the deformation at one of the two block faces is to be set to zero (as there is no predefined deformation to account for) $\Delta x_{i_{\min }, j, k}$ or $\Delta x_{i_{\max }, j, k}$ are set to zero respectively. The displacement $\Delta x_{[\mathrm{i}]}$ is applied, resulting in a grid representing an intermediate deformation state. The deformation in $j$ - and $k$-direction is applied in the same way, based on the intermediate grid obtained from the preceding deformation process:

$$
\begin{gathered}
\Delta x_{[\mathbf{j}], i, j, k}=\Delta x_{i, j_{\min }, k} \cdot\left(1-3 g_{[\mathbf{j}] i, j, k}^{2}+2 g_{[\mathbf{j}] i, j, k}^{3}\right)+\Delta x_{i, j_{\max }, k} \cdot\left(3 g_{[\mathbf{j}] i, j, k}^{2}-2 g_{[\mathbf{j}] i, j, k}^{3}\right) \\
\Delta x_{[\mathbf{k}], i, j, k}=\Delta x_{i, j, k_{\text {min }}} \cdot\left(1-3 g_{[\mathbf{k}] i, j, k}^{2}+2 g_{[\mathbf{k}] i, j, k}^{3}\right)+\Delta x_{i, j, k_{\max }} \cdot\left(3 g_{[\mathbf{k}] i, j, k}^{2}-2 g_{[\mathbf{k}] i, j, k}^{3}\right)
\end{gathered}
$$

The faces of the grid finally obtained conincide with the prescribed cut face coordinates. This is due to the fact that the deformation process in $j$-direction conserves the $i=i_{m i n^{-}}$and $i=i_{\text {max }}$-face coordinates previously created by the deformation process in $i$-direction. This can be directly seen from the fact that e.g. the $i=i_{m i n}$-edge at the $j=j_{\text {min }}$ block face matches the $j=j_{\text {min }}$-edge at the $i=i_{\text {min }}$ block face. Hence, $\Delta x_{[\mathbf{j}], i_{\text {min }}, j, k}$ is identical to zero. One can show that the sequence of deformations along the different index directions is invariant to the result. Hence the method generates a unique deformed state of the grid. The deformation of the blocks not contributing to the surface is illustrated by the blue blocks in Figure 1(b) and (c).

The deformation tool obtained from the above deformation strategy proved to work well on a variety of grid topologies. Furthermore the algorithm is robust and extremely fast. Compared to our previous monoblock tool we are now able to use much more convenient blade grid topologies and hence to achieve a better convergence of the flow computation. The implementation of the deformation tool in FLOWer has been parallelized as far as possible leading to an additional performance gain.

In case of a Chimera computation the outer mesh boundaries of the blade grid structure do not necessarily need to remain fixed in space relative to the rotating system. Thus, in order to minimize the deformation to be handled by the deformation tool, the grid deformation process is carried out relative to the blade secant system connecting blade root and blade tip in deformed state. Compared to the rotating rotor hub system the deformation amplitudes can be significantly reduced. The kinematic state of the blade relative to its secant system is taken into account by a rigid body transformation of the complete grid system.

\section{Weak Coupling Strategy}

\section{A. Trim Objectives and Control Inputs}

As already stated earlier the trim procedure is restricted to the main rotor of the configuration. This means a predefined trim state (e.g. a prescribed level of time-averaged loads) is only achived for the main rotor and not for the full helicopter. Aerodynamic loads on other components of the configuration besides the main rotor are not yet taken into account for the coupling process. This procedure is wellsuited for the reproduction of experimental conditions as in most cases only the main rotor and not the full configuration is trimmed towards a certain trim state. This will also be the case for the GOAHEAD campaign.

The actual trim ojectives may be defined in different ways. If the numerical simulation results are to be compared to experimental data the trim objectives are given from the experiment. Mostly the experimental rotor will be trimmed towards a certain time-averaged global loading. It is quite evident that the number of prescribed trim objectives defines the number of control inputs to set free. One of the most common approaches is to set collective and cyclic pitch angles free and to prescribe the timeaveraged global rotor thrust, the global rotor rolling moment and the global rotor pitching moment. This trim law has e.g. been applied to the HART and HART-II test campaigns [31]. It has the advantage that the trim objectives correspond almost directly to the the input control angles, i.e. cross-correlations are small.

For the GOAHEAD project it was decided to apply a pure force trim, i.e. to predefine the rotor vertical, lateral and longitudinal force. Hence these trim objectives are also applied to the numerical simulations of the present paper. According to the experimental procedure the collective and cyclic pitch angles are used as free control inputs and the rotor shaft angle will be held fixed at the experimental value. 


\section{B. Coupling and Trim Procedure}

The idea of the weak coupling scheme is as follows: HOST uses CFD loads to correct its internal 2D aerodynamics and re-trims the rotor. The blade dynamic response is introduced into the CFD calculation in order to obtain updated aerodynamic loads. This cycle is repeated until the CFD loads match the blade dynamic response evoked by them. A criterion for this converged state is given by the change of the control inputs with respect to the preceding cycle. Convergence has been reached after the changes in the free controls have fallen below an imposed limit. The specific steps of the coupling procedure are thus given as follows:

1. HOST determines an initial trim of the rotor based on its internal 2D aerodynamics derived from airfoil tables. The complete blade dynamic response for a given azimuth angle is fully described by the modal base and the related generalized coordinates.

2. The blade dynamic response is taken into account in the succeeding CFD calculation by the reconstruction of the azimuth angle dependent blade deformation from the modal base and by the respective grid deformation of the blade grid.

3. The CFD calculation determines the 3D blade loads in the rotating rotor hub system $\left(F_{x}[N / m]\right.$, $\left.F_{y}[N / m], F_{z}[N / m], M_{x}[N m / m], M_{y}[N m / m], M_{z}[N m / m]\right)$ for every azimuth angle and radial section of the blade.

4. For the next trim HOST uses a load given by

$$
\bar{F}_{H O S T}^{n}=\bar{F}_{2 D}^{n}+\bar{F}_{3 D}^{n-1}-\bar{F}_{2 D}^{n-1}
$$

$\bar{F}_{2 D}^{n}$ represents the free parameter for the actual HOST trim. A new dynamic blade response is obtained which is expressed by an update of the generalized coordinates.

5. Steps (2) to (4) are repeated until convergence has been reached, i.e. when the difference

$$
\Delta \bar{F}^{n}=\bar{F}_{2 D}^{n}-\bar{F}_{2 D}^{n-1} \longrightarrow 0
$$

tends to zero and the trim-loads depend only on the 3D CFD aerodynamics.

It has not yet been defined which components of the loads are actually modified by CFD aerodynamics during the HOST re-trim in step (4). The most general approach is to correct all six force and moment components of each spanwise blade section in HOST. In this case $\bar{F}$ is given by $\bar{F}=\left(F_{x}, F_{y}, F_{z}, M_{x}, M_{y}, M_{z}\right)^{T}$. However the blade dynamics is dominated by the distribution of spanwise thrust $\left(F_{z}\right)$, drag $\left(F_{y}\right)$ and pitching moment $\left(M_{x}\right)$ which directly affect the blade bending and torsion. The influence of $F_{x}, M_{y}$ and $M_{z}$ can be considered as negligible. It has thus proven to correct only $F_{z}, F_{y}$ and $M_{x}$ by CFD aerodynamics. This procedure has also been used for the computations presented in the paper.

The weak coupling method enforces the periodicity of the solution as on the one hand the blade dynamics are provided to the CFD method as Fourier series of the modal base and on the other hand a Fourier decomposition of the CFD loads is performed before providing them to the trim module of the CSD method. It is mandatory that the updated CFD loads for each successive trim are periodic with respect to the azimuth angle. After the CFD calculation has been restarted from the previous run, a certain number of time steps (i.e. a certain azimuth angle range) is necessary until the perturbation introduced by the updated set of generalized coordinates has been damped down and a periodic answer is obtained again. Clearly, this state is reached more quickly, the smaller the initial disturbance. For this reason the azimuth angle range covered by the CFD calculation can be reduced with an increasing number of re-trims. The changes in the free controls and the blade dynamic response become smaller from one re-trim to the next.

\section{Data Exchange between CFD and CSD}

The data exchange between CFD and CSD is performed on the blade surface as the common interface between fluid and structure side: The dynamic state of the blade (blade articulation and deformation) has to be taken into account on for the CFD computation and the aerodynamic loads predicted by CFD have to be taken into account on CSD side for the rotor re-trim. As stated above the weak coupling scheme enforces the periodicity of the solution by using Fourier series both for the description of the blade dynamics and for the aerodynamic loads. It has thus to be defined how many harmonics are to be used 
on either sides. Furthermore, the current implementation allows for non-matching radial discretizations of the blade on fluid and structure side. Hence an interpolation process for the blade deformation on the CFD discretization and an interpolation process of the CFD sectional loads on the CSD discretization is performed.

\section{Data transfer from CSD to CFD}

HOST uses a modal approach for the description of the blade deformation. Hence, a certain number of modal shapes, together with the corresponding harmonic description of the weighting factor, are used to yield a deformation description. Both the number of modal shapes considered and the number of harmonics taken into account are user-specified. Due to structural damping the excitation of higher modes and higher harmonics can be considered as small. It is thus sufficient to use a limited number of modal shapes and harmonics for the CSD computation. The CSD model applied to the computations of the present paper features a radial discretization of 85 blade elements. We used a modal base consisting of the first 10 mode shapes and five harmonics for the description of the generalized coordinate.

During the CFD computation the blade deformation is reconstructed for the current azimuth angle from the modal description provided by HOST. This process includes the interpolation of the deformation from the CSD discretization onto the radial discretization of the blade mesh. Due to the consideration of only a limited number of mode shapes the deformation along the blade span is smooth. In addition, the radial resolution of the CFD grid is mostly finer than the CSD discretization. Hence the interpolation process from CSD onto the CFD grid is uncritical.

\section{Data transfer from CFD to CSD}

The data transfer from CFD to CSD is the more critical point. The CFD loads on the rotor blade are provided to HOST as distributed loads along the blade span. HOST performs an interpolation of the distributed loads onto its discretization in order to obtain discrete loads at each radial blade element. Due to the differing radial discretizations the conservativity of the data transfer is not exactly guaranteed. Hence small deviations both in the radial load distribution and in the overall integrated loading may be generated. This drawback could be eliminated by the exchange of discrete loads instead of distributed loads. However, this would require identical dicretizations on either sides or a special interpolation treatment.

Furthermore, it has to be clarified how many harmonics are to be used for the Fourier decomposition of the CFD loads. As stated above high frequency loads do not lead to a direct structural response due to damping. However, high frequency loads may also influence the low frequency structural response. Hence the number of harmonics considered is an essential point. For most configurations ten harmonics turned out to be sufficient to capture the azimuthal load distribution predicted from CFD with the required accuracy.

\section{Results and Discussion}

\section{A. Flight Conditions and Computational Setup}

The GOAHEAD setup represents a generic helicopter configuration consisting of the 7AD model main rotor (2.1 meter radius) including rotor head, the Bo105 model tail rotor and a NH90 fuselage. The configuration will be mounted on a pivoting sting in the closed test section of the DNW Large Low Speed Facility. The main rotor rotates clockwise seen from top and the tail rotor rotates upper side forward. Different flight conditions covering the typical helicopter flight regime will be measured in the forthcoming measurement campaign.

The present paper adresses three of the flight cases: The Low-Speed Pitch-Up, the High-Speed TailShake and the highly loaded rotor case. Low-Speed Pitch-Up occurs during transition of the helicopter from hover into forward flight and is characterized by an impingement of the main rotor wake with the horizontal stabilizer, leading to a change in the overall pitching moment. Tail-Shake is a wakeinduced vibration problem arising from interference between the wake of the main rotor hub and the tail part of the helicopter. The third test case is planned as a test case for Dynamic Stall. Dynamic Stall occurs on the retreating blade of a highly loaded rotor and represents a severe life-time problem of the pitch link rods. For the experimental test campaign the rotor loading actually realizable in the wind tunnel is limited due to blade stability restrictions and restrictions on the available rotor drive power. Preliminary investigations showed that the planned test condition is close to the occurance of Dynamic Stall and within the given drive power and blade loading limitations. 
For all GOAHEAD test cases a pure force trim of the main rotor will be performed. The flight conditions and the trim objectives are given in Table 1. As stated earlier the rotor is trimmed by adapting the collective and cyclic pitch control. The pitch angle of the configuration, and thus the rotor shaft angle, is held fixed at the experimental value.

\begin{tabular}{|l|c|c|c|}
\hline & LSPU & HSTS & HLDS \\
\hline Rotor RPM & 954 & 954 & 954 \\
\hline Blade Tip Mach Number $M a_{T i p}$ & 0.617 & 0.617 & 0.617 \\
\hline Free Stream Mach Number $M a_{\infty}$ & 0.059 & 0.204 & 0.259 \\
\hline Fuselage Pitch Angle $\alpha_{f}$ & $+5^{\circ}$ & $-2^{\circ}$ & $-7^{\circ}$ \\
\hline Rotor Shaft Angle $\alpha_{q}$ & $0^{\circ}$ & $-7^{\circ}$ & $-12^{\circ}$ \\
\hline Rotor Vertical Force $F_{z}$ & $4500 \mathrm{~N}$ (upward) & $4500 \mathrm{~N}$ (upward) & $6068 \mathrm{~N}$ (upward) \\
\hline Rotor Lateral Force $F_{y}$ & 0 & 0 & 0 \\
\hline Rotor Longitudinal Force $F_{x}$ & $-44 \mathrm{~N}$ (forward) & $-530 \mathrm{~N}$ (forward) & $-475 \mathrm{~N}$ (forward) \\
\hline
\end{tabular}

Table 1. Flight condition and trim objective

Two different CFD grid setups have been used for the computation and will be compared with respect to the coupled and trimmed rotor solution:

1. Isolated Rotor Setup: Grid setup consisting of the wind tunnel mesh (background grid) and the main rotor.

2. Full Configuration: Grid setup consisting of the wind tunnel mesh (background grid), the main rotor including rotor head, the fuselage, the tail rotor and the sting.

The grid data of the setups is summarized in Table 2. Figure 2 shows the full CFD grid configuration. In order to provide a comparable grid resolution of both setups in the vicinity of the rotor (and hence to provide comparable wake conservation properties) the background grid resolution of the isolated rotor setup has been refined accordingly.

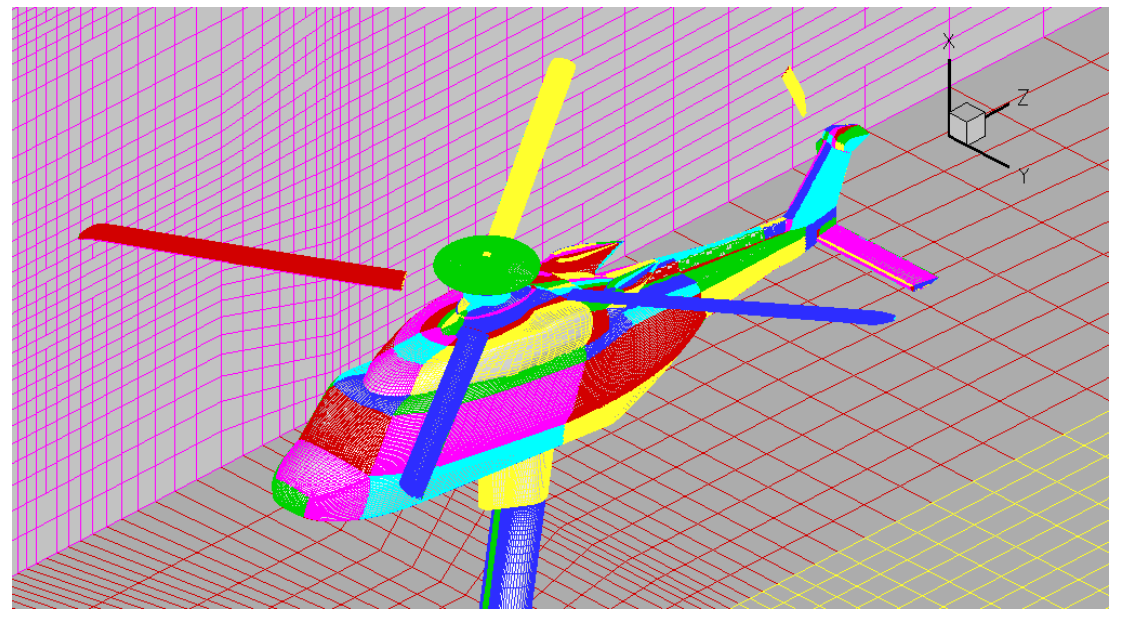

Figure 2. Full CFD grid configuration

All CFD computations have been performed with an azimuthal time step of $2^{\circ}$ for the main rotor, which is equivalent to an azimuthal time step of $10^{\circ}$ for the tail rotor. The computations have been run fully turbulent using the $k \omega$ turbulence model according to Wilcox.

In contrast to our previous computations the multi-block grid deformation tool allows for the usage of blade grid structures consisting of several blocks. The blade grid structures use a C-type topology in chordwise direction and a H-type topology in spanwise direction and consist of 10 blocks each. The blade surface is restricted to the aerodynamic part, i.e. the blade shaft and the attachment to the rotor hub is not modelled. Figure 3 shows the blade grid structure and the blade surface mesh. 


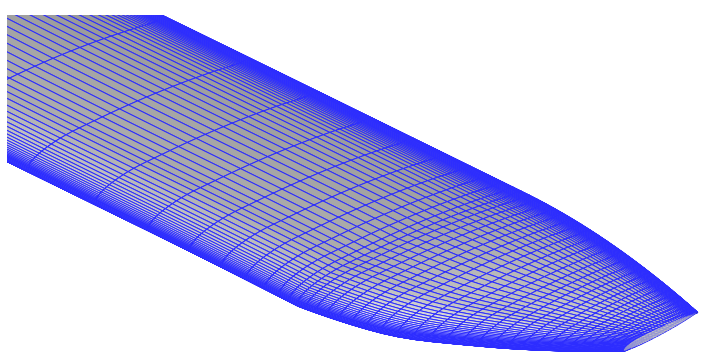

(a) Blade surface mesh

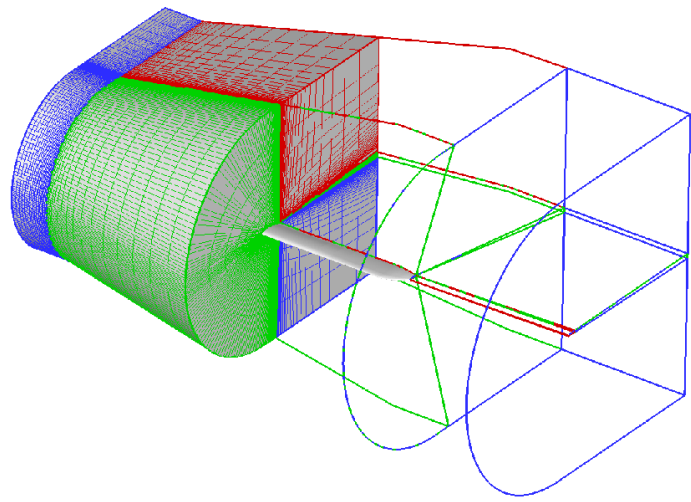

(b) Blade grid structure

Figure 3. Blade surface mesh and block structure

\begin{tabular}{|l|c|c|}
\hline & Isolated Rotor Setup & Full Setup \\
\hline Number of structures & 5 & 10 \\
\hline Number of blocks & 66 & 169 \\
\hline No. of cells main rotor & $4 \times 811,200$ & $4 \times 811,200$ \\
\hline No. of cells background & $2,845,440$ & 265,728 \\
\hline No. of cells fuselage & - & $8,694,848$ \\
\hline No. of cells tail rotor & - & $2 \times 335,488$ \\
\hline No. of cells rotor head & - & 830,208 \\
\hline No. of cells strut & - & 476,672 \\
\hline Total number of cells & $6,090,240$ & $14,183,232$ \\
\hline
\end{tabular}

Table 2. Grid data

\section{B. Low-Speed Pitch-Up}

Taking the LSPU test case as an example, we will show the convergence properties of the weak coupling scheme in more detail than for the other two test cases. The convergence of the control angles $\theta_{0}, \theta_{C}$ and $\theta_{S}$ versus the coupling iterations is shown in Figure 4. The Figure shows both the convergence of the isolated rotor computation and the convergence of the complete helicopter configuration. Note that a preliminary investigation utilizing a coarsened grid system has been carried out for the complete helicopter. The trim iterations 0 to 5 correspond to this reduced grid system. After trim iteration 5 we switched to the full grid system given in Table 2. A converged state is obtained after four re-trims in case of the isolated rotor, and after 10 trim trim iterations for the complete configuration (only the last five iterations correspond to the full grid system). In converged state the changes in all three control angles have dropped below $0.03^{\circ}$. One can see that the weak coupling technique works for the full configuration with the same quality as for the isolated rotor.

Figures 5 and 7 show the development of the unsteady global rotor forces versus the coupling iterations for the isolated rotor and the complete configuration, respectively. A trimmed state is reached after nine rotor revolutions of the isolated rotor. In case of the complete configuration the initial CFD computation of the 0th trim has been extended to six rotor revolutions in order to make sure that a periodic behaviour has actually been reached. A trimmed state is obtained after twelve rotor revolutions. Figures 6 and 8 show the corresponding mean values of the CFD loads for the last quarter revolution of each trim cycle. The Figures prove that the prescribed trim condition is actually reached with a sufficient accuracy. As is can be seen from the Figures the mean values do not exactly coincide with the the trim value prescribed on HOST side. This is likely to be caused by the fact that the data exchange between CFD and CSD is not strictly conservative. The small deviation from the actual objective (approximately $0.5 \%$ for the vertical force) is still acceptable.

Table 3 summarizes the rotor control angles in trimmed state for both grid configurations. The table 


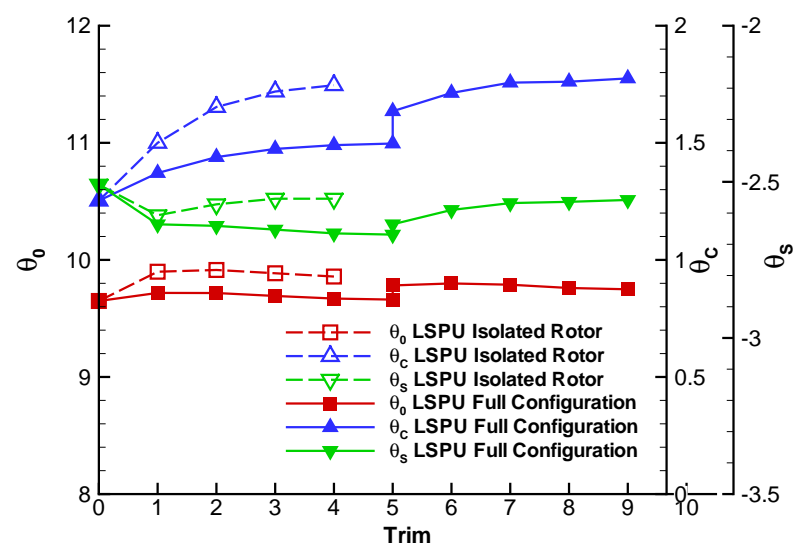

Figure 4. LSPU trim convergence of control angles

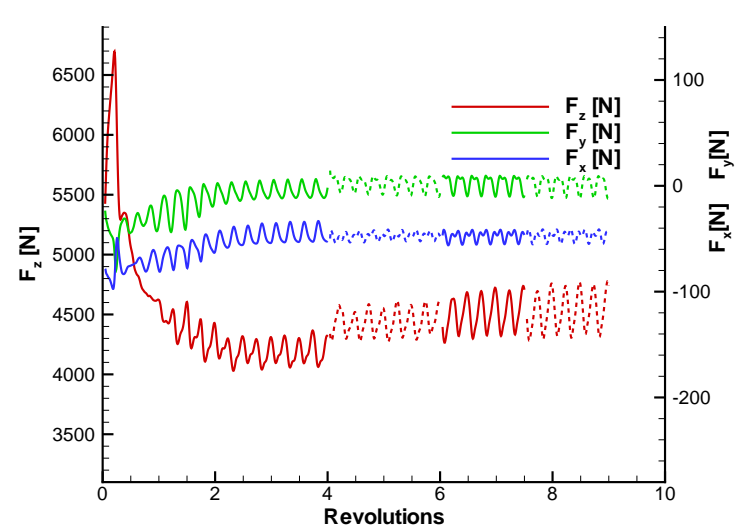

Figure 5. LSPU time history of unsteady CFD loads (isolated rotor)

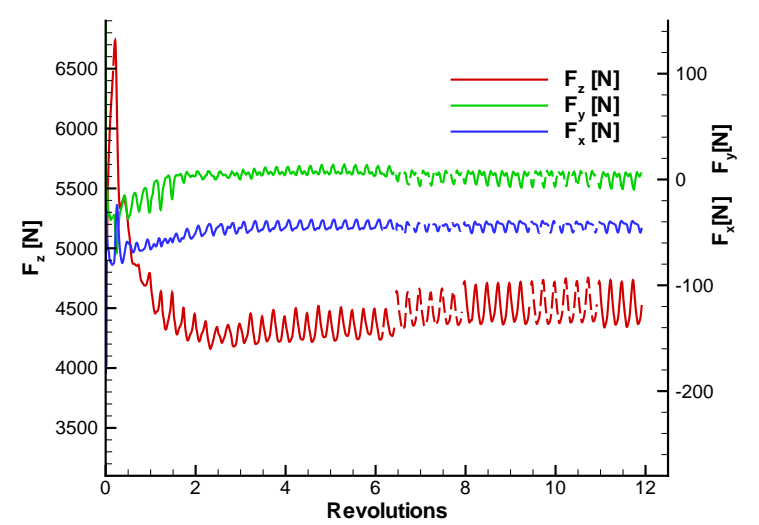

Figure 7. LSPU time history of unsteady CFD loads (complete helicopter)

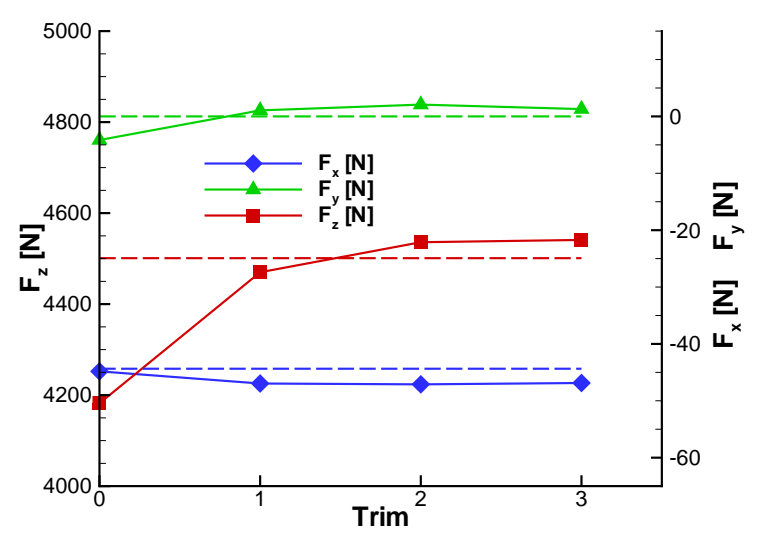

Figure 6. LSPU trim history of CFD mean values (isolated rotor)

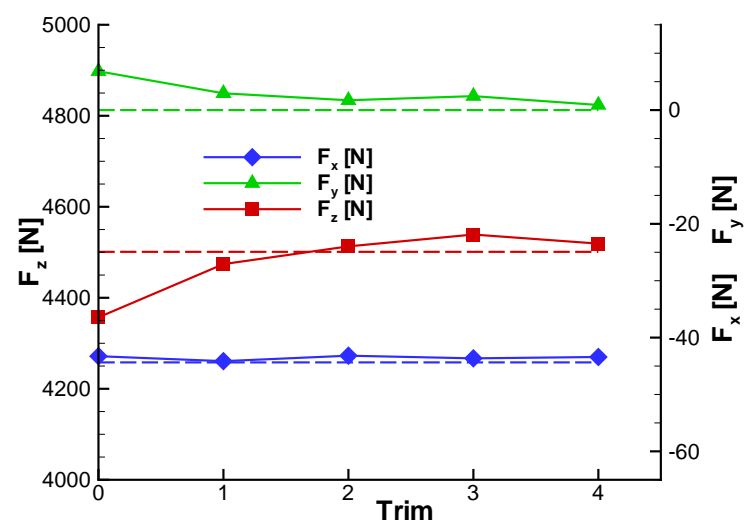

Figure 8. LSPU trim history of CFD mean values (complete helicopter) 


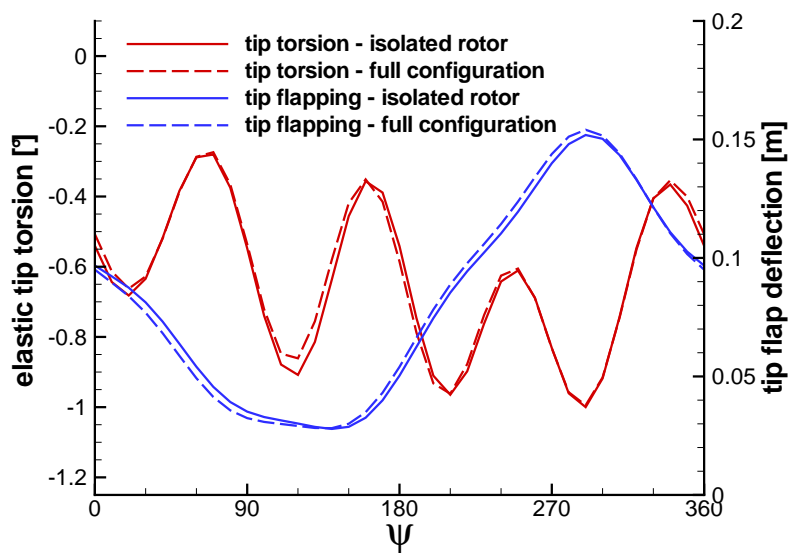

Figure 9. Comparison of blade dynamic response, LSPU

includes the pitch control angles as well as the mean and 1/rev contributions to the articulations in the flap and lead-lag hinges. The study reveals only very small differences between the isolated rotor computation and the complete helicopter computation.

\begin{tabular}{|l|c|c|c|c|c|c|c|c|c|}
\hline & $\theta_{0}$ & $\theta_{C}$ & $\theta_{S}$ & $\beta_{0}$ & $\beta_{C}$ & $\beta_{S}$ & $\delta_{0}$ & $\delta_{C}$ & $\delta_{S}$ \\
\hline Isolated Rotor & 9.858 & 1.746 & -2.554 & -1.960 & -0.916 & 1.204 & 0.719 & 0.186 & -0.004 \\
\hline Complete Helicopter & 9.749 & 1.775 & -2.558 & -1.960 & -0.759 & 1.288 & 0.742 & 0.203 & -0.014 \\
\hline
\end{tabular}

Table 3. LSPU rotor control angles

The effect of the remaining helicopter components on the blade dynamics is illustrated in Figure 9 . The Figure compares the blade tip flapping amplitude and the blade tip torsion distribution of the isolated rotor and the complete helicopter configuration in trimmed state. Only negligible differences between the configurations are noticed. As both trim control angles and blade dynamics are virtually not influenced by the consideration of the additional helicopter components, the influence on the aerodynamic loading on the rotor disk should be minor, too. This is proven by Figures 10 and 11, showing the thrust and drag distribution on the rotor disk. In both Figures (a) shows the distribution of the isolated rotor, (b) shows the distribution of the complete helicopter, and (c) gives the delta between complete configuration and isolated rotor. Positive values of $\Delta F_{z}$ correspond to a higher thrust of the complete configuration compared to the isolated rotor. Note that the circumferential in-plane force $F_{y}$ is positive when pointing from the trailing to the leading edge. Hence positive values correspond to an in-plane propulsive force, and negative values correspond to drag. Correspondingly positive values of $\Delta F_{y}$ denote a drag reduction of the complete configuration compared to the isolated rotor.

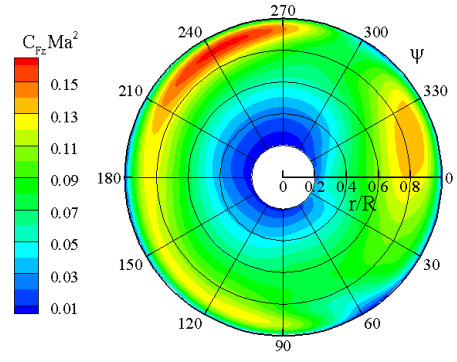

(a) Isolated Rotor

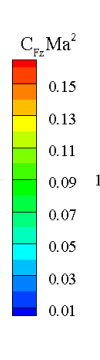

(b) Full Configuration

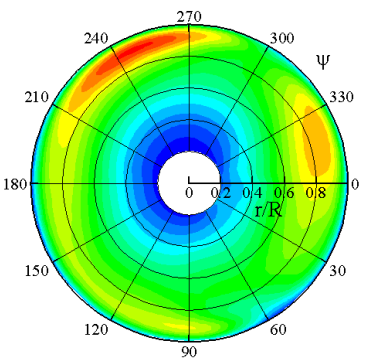

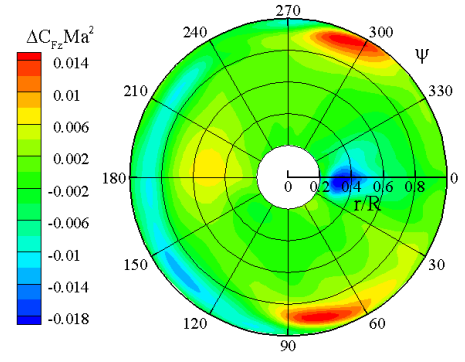

(c) Delta between Full Configuration and Isolated Rotor

Figure 10. LSPU thrust distribution on rotor disk

Altough both the overall thrust and drag distribution of both configurations are almost identical local differences can be spotted in the delta plots of Figures 10(c) and 11(c). The rotor of the full configuration 


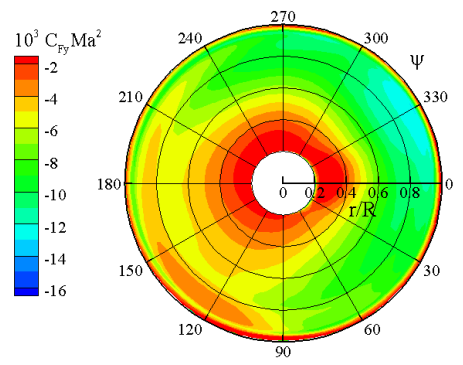

(a) Isolated Rotor

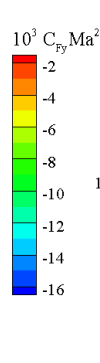

(b) Full Configuration

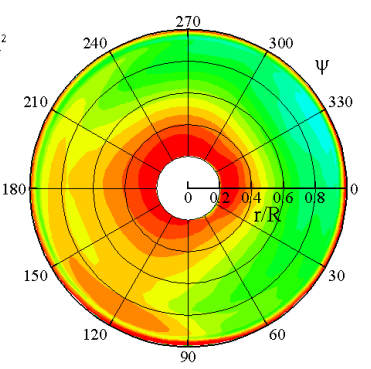

(c) Delta between Full Configuration and Isolated Rotor

Figure 11. LSPU drag distribution on rotor disk

produces less lift in an area from $r / R=0.85$ to $r / R=0.95$ on the front part of the rotor disk and in the region of $r / R=0.2$ to $r / R=0.4$ around $\psi=0^{\circ}$. Higher lift is produced in the blade tip region around $\psi=60^{\circ}$ and $\psi=300^{\circ}$.

The lift decrease in the inboard radial region around $\psi=0^{\circ}$ is caused by interaction with the complex geometry of the engine fairing and the tail boom. This becomes apparent from Figure 12 which shows slices of the flow field at $Y=0$ for the isolated rotor configuration and the full configuration. The absolute value of the in-plane velocity vector, normalized by the far field velocity, is plotted as contour variable. From Figure 12(b) a separated flow region can be identified in the wake region of the engine casing below the inboard region of the aft blade. The streamline plot reveals a vortical structure which is not present for the isolated rotor configuration. Furthermore the Figure shows a low velocity region above the inboard blade region immediately behind the rotor head. Summarizing, the disturbed flow at the inboard blade region leads to the loss in thrust observed in Figure 10(c).

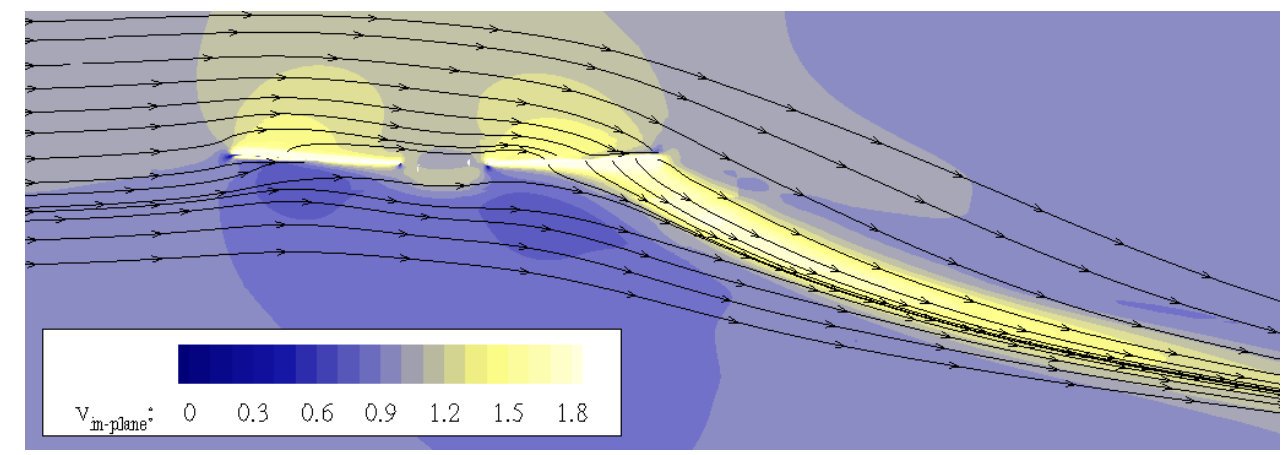

(a) Isolated Rotor

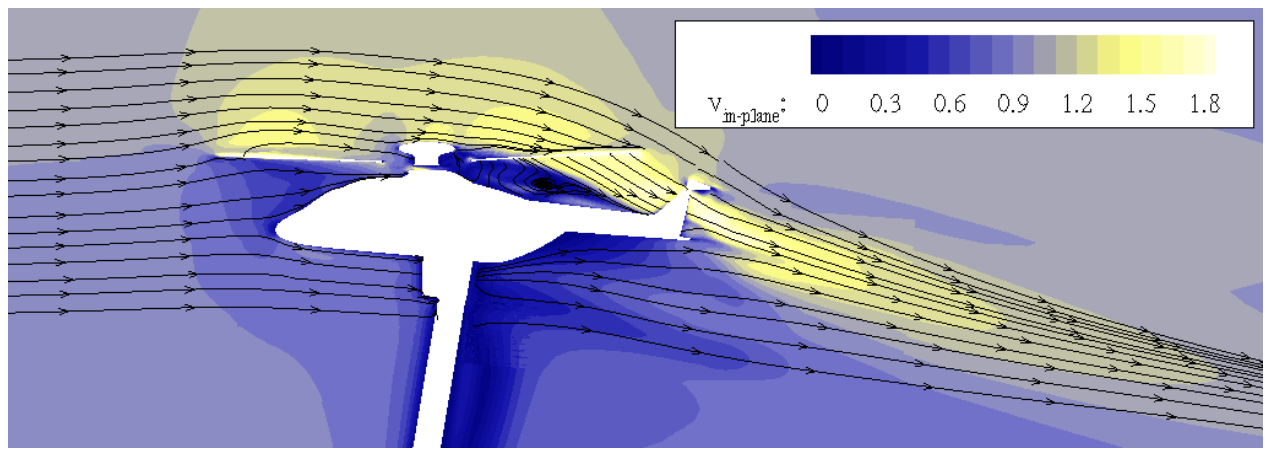

(b) Full Configuration

Figure 12. Flow field in $\mathrm{Y}=0$ plane

The thrust reduction comes along with an increase in drag as clearly shown from the red spot in Figure 11(c). Compared to the isolated rotor the drag distribution of the full configuration features regions of increased and reduced drag. Thus the overall effect on the rotor power consumption is hard to determine from this representation. One would expect an increased power consumption for the full 
configuration. However the integration of the total rotor torque shows that this is actually not the case: A power requirement of $51.08 \mathrm{~kW}$ is obtained for the full configuration whereas the power requirement of the isolated rotor is slightly higher at $51.84 \mathrm{~kW}$. This finding comes against expectation because it predicts a reduction in power consumption when interference between the rotor and the rest of the helicopter takes place. Apparently the minor changes in the thrust distribution, caused by interference of the main rotor flow with the fuselage and the rotor head, lead to a slightly improved Figure of Merit. A thorough explanation is however difficult to make in view of the multitude factors involved.

\begin{tabular}{|l|c|c|}
\hline & Isolated Rotor & Complete Helicopter \\
\hline Rotor power consumption & $51.84 \mathrm{~kW}$ & $51.08 \mathrm{~kW}$ \\
\hline
\end{tabular}

Table 4. Rotor power consumption in LSPU

Figure 13 compares the 3D flow field of both configurations. The vortex system is visualized using the $\lambda_{2}$-criterion of Jeong and Hussain [16]. The comparison of 13(a) and (b) shows that the tip vortex system is not significantly changed by the presence of rotor head, fuselage and tail rotor. The location of the distinct tip vortices shed from the rear part of the rotor disk does barely change, except from the fact that the vortices of the full configuration are chopped up by the tail rotor. The major characteristics of the wake are thus similar which is also substantiated by the contour plot given in Figure 12. Things look different for the inboard wake structure. In case of the isolated rotor the blade inboard wake is convected downstream unhamperedly whereas in case of the full configuration the flow field is dominated by the interaction between blade inboard wake, rotor head wake and fuselage. Figures 13 (b) and 12 substantiate that the investigated flight condition is actually characterized by the occurance of the Pitch-Up phenomenon. Both Figures show that the horizontal stabilizer is likely to be located within the rotor downwash.

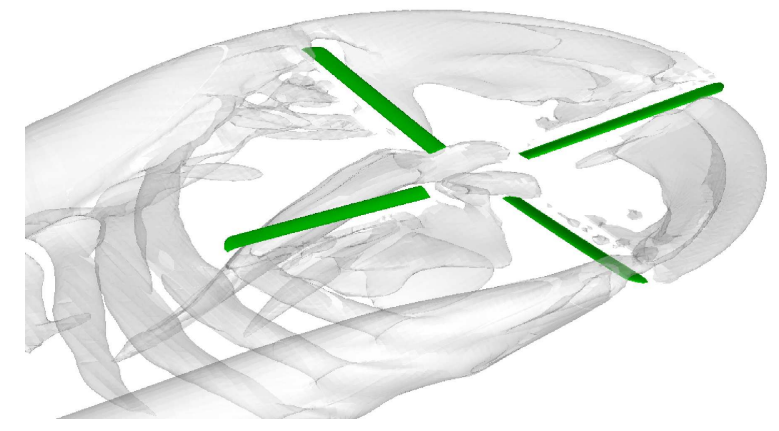

(a) Isolated Rotor

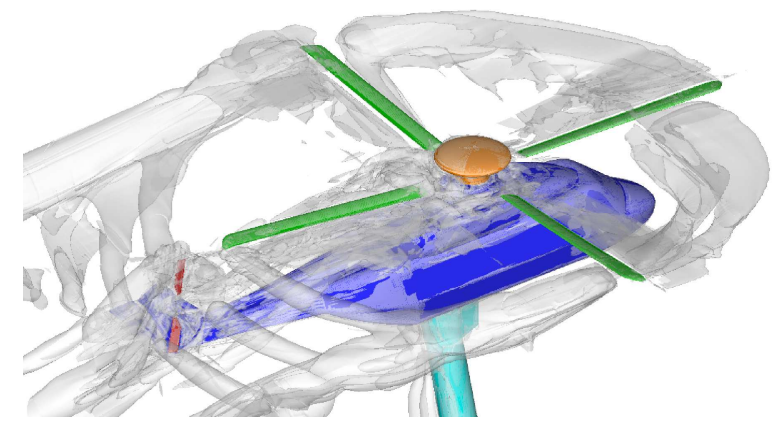

(b) Full Configuration

Figure 13. Visualiziation of 3D flow field using $\lambda_{2}$ criterion, LSPU

\section{High-Speed Tail-Shake}

The trim convergence of the control angles $\theta_{0}, \theta_{C}$ and $\theta_{S}$ for the HSTS test case versus the coupling iterations is given from Figure 14. Again, the trim computation of the complete configuration was started with a coarsened grid system (trim iterations 1 to 4 ). Iterations 4 to 7 refer to the full grid system. No significant changes between the control angles of the complete system and the ones obtained for the isolated rotor are observed. The rotor trim of the complete configuration reveales a slightly higher collective pitch (approximately $0.15^{\circ}$ ) and a slightly higher lateral cyclic pitch (approximately $0.17^{\circ}$ ). The longitudinal cyclic pitch is barely influenced.

Table 5 shows that noticable changes are obtained in the lateral and longitudinal flapping amplitudes while the constant part of the blade flapping and the blade lag angles remain almost unchanged. However one has to take into account that $\beta_{C}$ and $\beta_{S}$ refer to the angular offset in the flap hinge. The angular change in the flap hinge is not a direct indication for the tip path plane angle as the elastic flapping deformation of the blades needs to be superimposed. The blade tip flapping distribution of Figure 15(a) represents a superposition of both effects (articulation and deformation) and thus provides a better measure of the effect of the remaining helicopter components on the tip path plane orientation. One can see that the azimuthal distribution reveals some differences between the isolated rotor and 


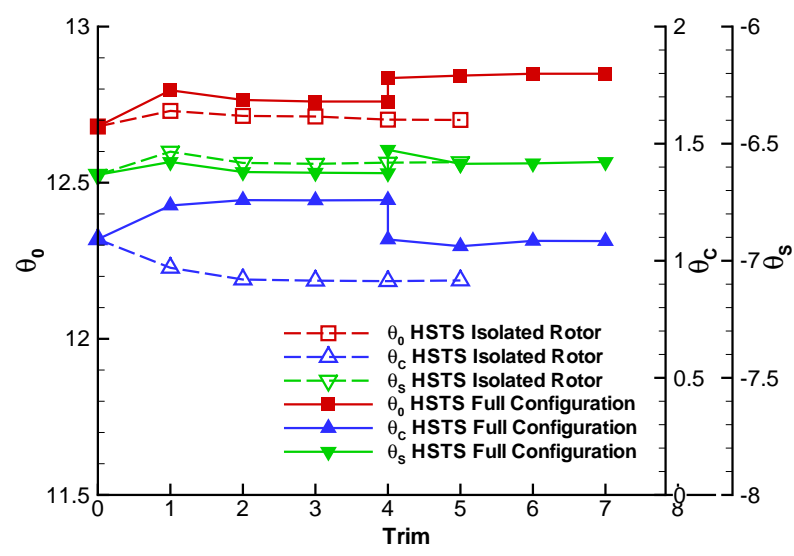

Figure 14. HSTS trim convergence of control angles

the complete configuration especially on the second half of the revolution. The distribution of the complete configuration is strongly $1 / \mathrm{rev}$ dominated. The distribution of the isolated rotor shows a larger contribution of higher-harmonic portions resulting in the reduced flapping amplitude around $\psi=270^{\circ}$.

A comparison of the blade tip torsion prediction is given in Figure 15(b). Both torsional distributions clearly show a $4 / \mathrm{rev}$ dominated behaviour. However the phase of the curves differs by approximately $\Delta \psi=30^{\circ}$ and the distribution of the isolated rotor computation reveals higher torsional amplitudes. An explanation for the altered blade dynamic behaviour might be deducted from the rotor disk loading, which will be considered in the following.

\begin{tabular}{|l|c|c|c|c|c|c|c|c|c|}
\hline & $\theta_{0}$ & $\theta_{C}$ & $\theta_{S}$ & $\beta_{0}$ & $\beta_{C}$ & $\beta_{S}$ & $\delta_{0}$ & $\delta_{C}$ & $\delta_{S}$ \\
\hline Isolated Rotor & 12.701 & 0.916 & -6.579 & -1.953 & -0.4 & 0.474 & -0.323 & 0.109 & -0.071 \\
\hline Complete Helicopter & 12.849 & 1.084 & -6.578 & -1.951 & -0.192 & 1.134 & -0.306 & 0.205 & -0.086 \\
\hline
\end{tabular}

Table 5. HSTS rotor control angles

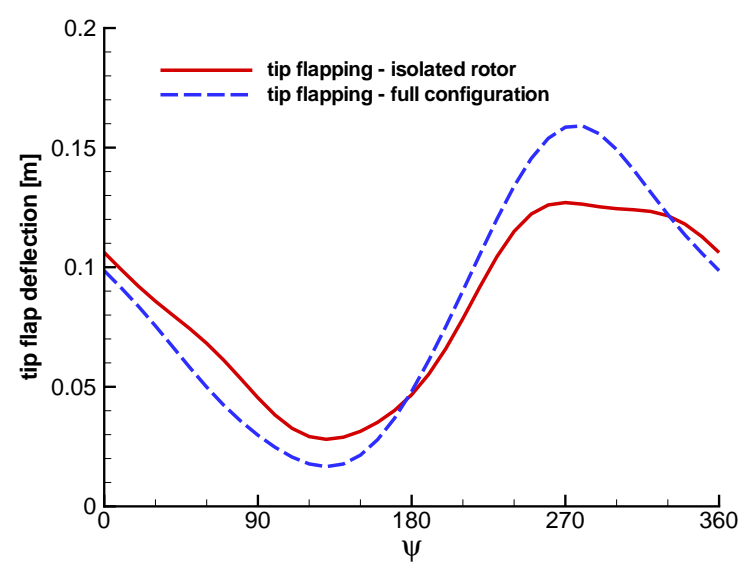

(a) Tip flapping motion

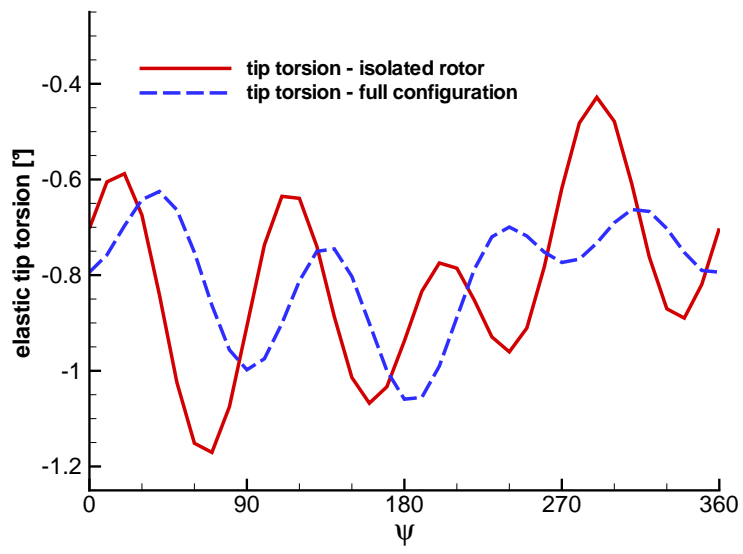

(b) Elastic tip torsion

Figure 15. Comparison of blade dynamic response, HSTS

The effect of the presence of the additional helicopter components on the rotor thrust distribution is presented in Figure 16. Both the thrust distribution of the isolated rotor and of the complete configuration is characterized by high load zones in the blade outboard region of the blade in the rear and front part of the rotor disk. Negative loading is found at the blade tip region between $90^{\circ}$ and $120^{\circ}$ and at the inboard region of the retreating blade. Figure 16(c) shows the change in the rotor thrust distribution between 
both configurations. As both the isolated rotor and the rotor of the complete helicopter configuration were trimmed towards the same vertical force, the integral effect of the thrust redistribution is close to zero. Local differences are spotted around $\psi=0^{\circ}$ and $\psi=180^{\circ}$, and around $30^{\circ}<\psi<120^{\circ}$. The changes around $\psi=0^{\circ}$ and $\psi=180^{\circ}$ can be directly attributed to the fuselage: The upward deflection of the flow over the fuselage front part increases the effective angle of attack and thus leads to a thrust increase around $\psi=180^{\circ}$. The thrust decrease around $\psi=0^{\circ}$ can be related to the interaction with the rotor head wake as it becomes apparent from the wake visualization given in Figure 18.

The differences observed around $30^{\circ}<\psi<120^{\circ}$ are harder to explain. One would expect to observe the strongest effects on the load distribution in the vicinity of the fuselage. However Figure 16 reveals that this is not the case and differences are obtained throughout the entire blade radius on the advancing blade side. This phenomenon can only be attributed to the fact that the presence of the fuselage leads to changes in the entire rotor flow field and induced velocity distribution. Due to the coupled nature of the problem this involves changes to the blade dynamics response as well, as already verified from Figure 15 .

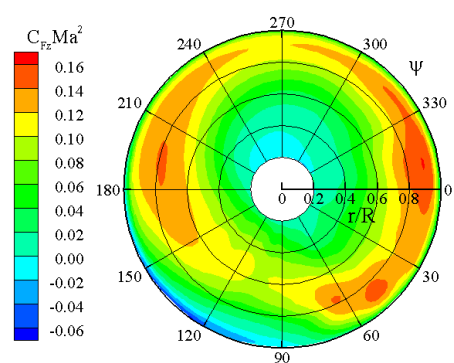

(a) Isolated Rotor

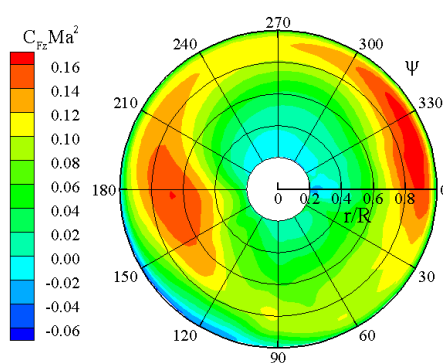

(b) Full Configuration

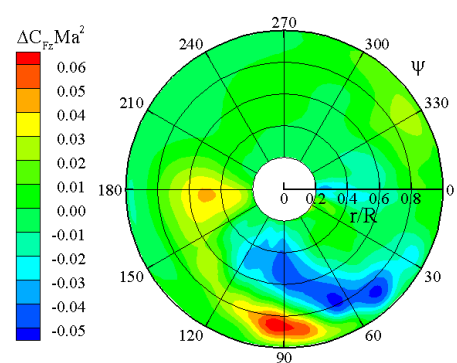

(c) Delta between Full Configuration and Isolated Rotor

Figure 16. HSTS thrust distribution on rotor disk

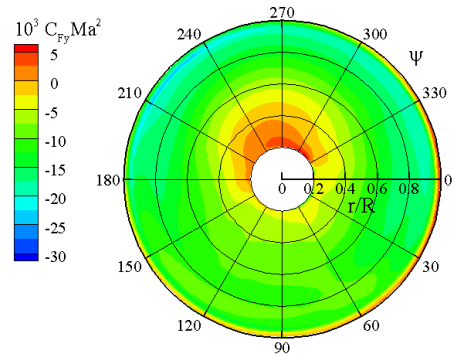

(a) Isolated Rotor

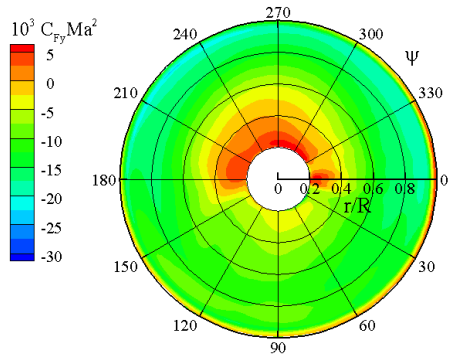

(b) Full Configuration

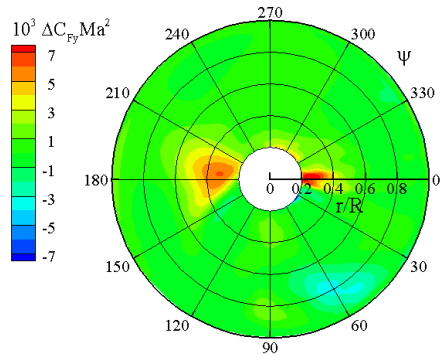

(c) Delta between Full Configuration and Isolated Rotor

Figure 17. HSTS drag distribution on rotor disk

Although both the isolated rotor and the rotor of the complete helicopter were trimmed towards an identical trim objective, the changes in the blade dynamics and the aerodynamic loading might affect the power consumption of the rotor. A measure of the power consumption is the drag distribution (i.e. the in-plane circumferential force distribution) on the rotor disc. The drag distribution of both configurations is compared in Figure 17. As already noticed for the LSPU case, the drag distribution of the full configuration features regions of increased and reduced drag compared to the isolated rotor. The largest differences are observed in the inboard region around $\psi=0^{\circ}$, where the thrust increase is related to a drag increase, and around $\psi=180^{\circ}$, where the drag increase can be explained from the interaction with the rotor head wake. Remarkably, the deviations in the thrust distribution on the advancing blade side do not lead to significant changes in the drag distribution. An evaluation of the rotor power consumption in trimmed state reveals a rotor power of $85.10 \mathrm{~kW}$ for the isolated rotor, and a rotor power of $84.41 \mathrm{~kW}$ for the complete configuration. Again, the power requirement of the isolated rotor is slightly higher. However the difference is small.

Concluding, one can say that local changes both in the distribution of aerodynamic loads and in the blade dynamic response were detected for the HSTS flight case. A straightforward explanation for the altered torsional behaviour and the changes in blade loading on the advancing blade side can not be 


\begin{tabular}{|l|c|c|}
\hline & Isolated Rotor & Complete Helicopter \\
\hline Rotor power consumption & $85.10 \mathrm{~kW}$ & $84.41 \mathrm{~kW}$ \\
\hline
\end{tabular}

Table 6. Rotor power consumption in HSTS

given due to the multitude factors involved. However, both aerodynamic and dynamic effects were found not pronounced enough to lead to a significant change in the overall rotor behaviour and rotor power consumption.

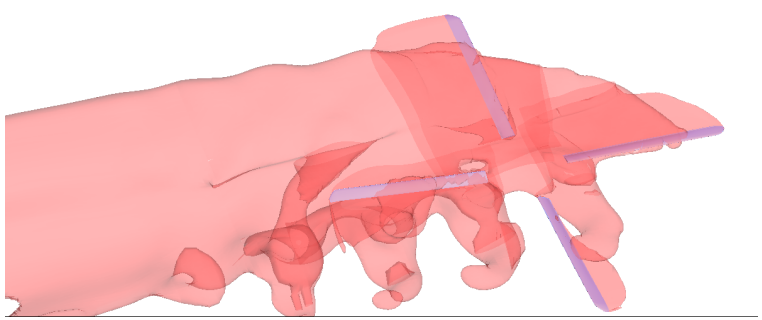

(a) Isolated Rotor

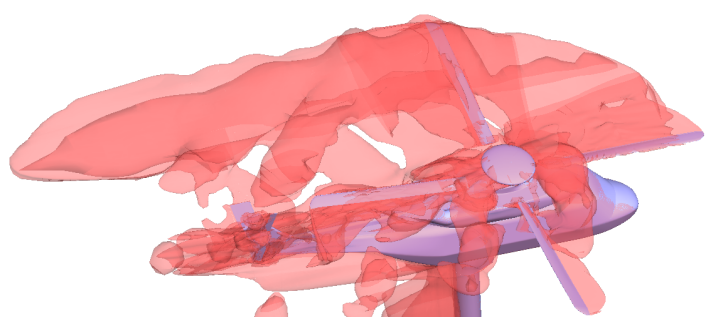

(b) Full Configuration

Figure 18. Visualiziation of 3D flow field using iso-entropy surfaces, HSTS

\section{Highly loaded rotor in Dynamic Stall}

The trim convergence of the control angles $\theta_{0}, \theta_{C}$ and $\theta_{S}$ for the HLDS test case versus the coupling iterations is given from Figure 19. Note that for this test case the computation of the complete helicopter comfiguration was directly started using the full grid system and the coarsened grid system was not used for initial trim computations. A trimmed state is obtained after four trim iterations for both the isolated rotor and the complete configuration. Due to the high rotor loading the collective setting has significantly increased compared to the HSTS case and reaches approximately $18^{\circ}$. The rotor of the complete configuration requires a slightly higher collective setting (approx. $0.3^{\circ}$ ). Both $\theta_{C}$ and $\theta_{S}$ differ about $0.3^{\circ}$ from the settings of the isolated rotor.

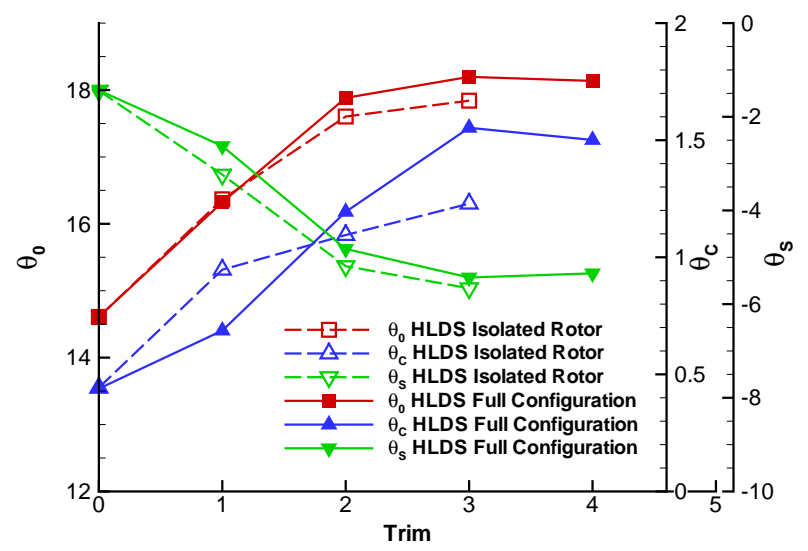

Figure 19. HLDS trim convergence of control angles

As for the HSTS case Table 7 reveals a similar mean flap angle for both cases, but deviations in the lateral and longitudinal flapping amplitudes. Both cases feature a very high value of the longitudinal flapping amplitude $\beta_{C}$. The value of $6.0^{\circ}$ for the isolated rotor is even exceeded by the one the complete configuration, coming close to $7.5^{\circ}$. As a consequence a very high inclination angle of the tip path plane is produced which manifests in blade tip flapping amplitudes of more than $0.35 \mathrm{~m}$ (see Figure 20(a)). Figure 21 supports these findings by showing the blade flapping state for the four azimuth angles $\psi=0^{\circ}, \psi=90^{\circ}, \psi=180^{\circ}$ and $\psi=270^{\circ}$. The black line denotes the reference plane perpendicular to the shaft. Note the large tip flap deflection at $\psi=180^{\circ}$. 


\begin{tabular}{|l|c|c|c|c|c|c|c|c|c|}
\hline & $\theta_{0}$ & $\theta_{C}$ & $\theta_{S}$ & $\beta_{0}$ & $\beta_{C}$ & $\beta_{S}$ & $\delta_{0}$ & $\delta_{C}$ & $\delta_{S}$ \\
\hline Isolated Rotor & 17.841 & 1.229 & -5.662 & -2.572 & 6.073 & 1.061 & -1.973 & 0.187 & 0.636 \\
\hline Complete Helicopter & 18.136 & 1.501 & -5.343 & -2.532 & 7.335 & 1.445 & -2.910 & 0.294 & -0.950 \\
\hline
\end{tabular}

Table 7. HLDS rotor control angles

Figure 20(b) compares the elastic blade tip torsion. The most significant changes are visible on the retreating blade side where elastic nose-down torsion of the complete configuration exceeds the one of the isolated rotor by approximately $0.5^{\circ}$. We will come back to this finding further below.

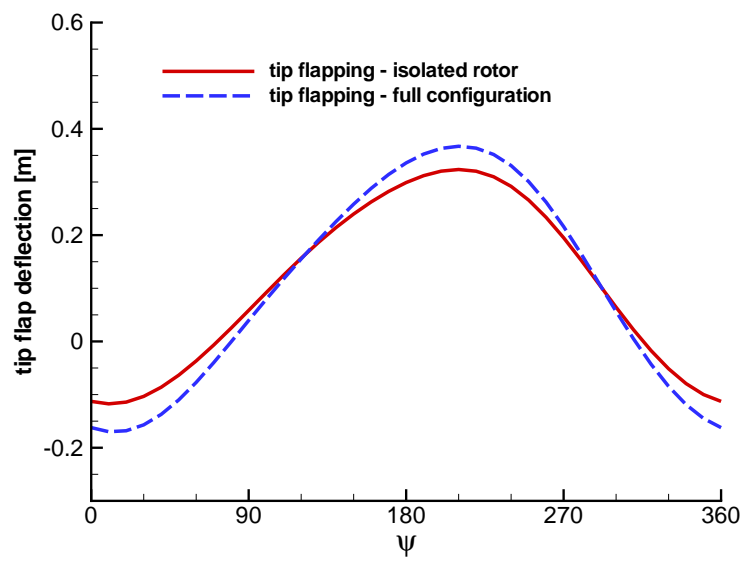

(a) Tip flapping motion

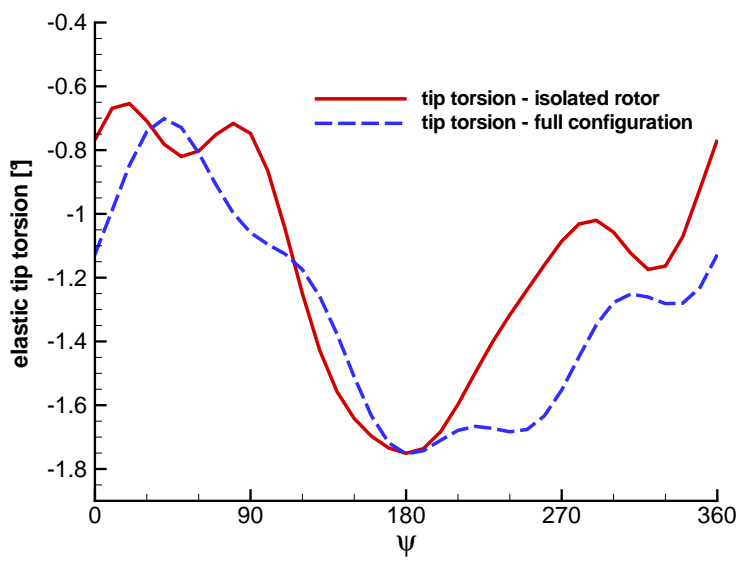

(b) Elastic tip torsion

Figure 20. Comparison of blade dynamic response, HLDS

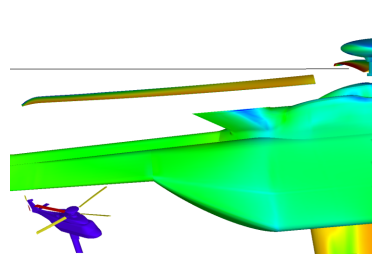

(a) $\psi=0^{\circ}$

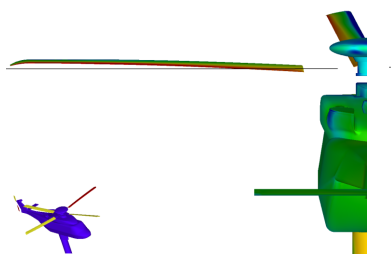

(b) $\psi=90^{\circ}$

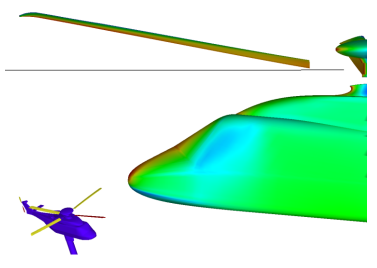

(c) $\psi=180^{\circ}$

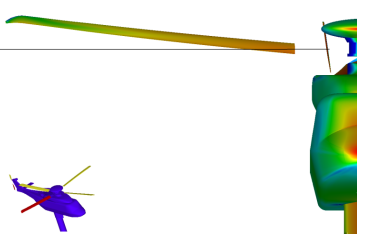

(d) $\psi=270^{\circ}$

Figure 21. Blade flapping response, HLDS

As already done for the two previous cases we will continue with the comparison of aerodynamic loading on the rotor disk. Figure 22 gives the thrust distribution on the disk and Figure 23 the drag distribution. In addition, the distribution of the sectional pitching moment distribution on the rotor disk is presented in Figure 24. Again, Figure 22(c) reveals local changes in the thrust distribution, which can partially be directly attributed to the fuselage, and whose integral effect is close to zero as both rotors have been trimmed towards an identical vertical force. The drag distribution shows regions of reduced drag and regions of increased drag. Reduced drag regions are located around $60^{\circ}<\psi<90^{\circ}$ and $180^{\circ}<\psi<210^{\circ}$. Regions of significantly increased drag are found in the outboard radial regions on the retreating blade side, starting at around $\psi=250^{\circ}$. An evaluation of the rotor power requirement reveals the remarkable result, that the power requirement of the isolated rotor of $130.00 \mathrm{~kW}$ has increased by more than $20 \%$ and reaches almost $160 \mathrm{~kW}$ (see Table 8). Recalling that a drag rise in the outboard radial region is most sensitive to rotor power this result is in line to the findings from Figure 23(c).

The question arises what mechanism causes the high increase in rotor power. As it becomes evident from Figure 24 the region of increased drag is connected to a region featuring a distict decrease in sectional pitching moment. Whereas no decrease in pitching moment is observed for the isolated rotor (Figure $24(\mathrm{a}))$, the complete configuration pitching moment distribution shows a decrease in the area of $240^{\circ}<$ 


\begin{tabular}{|l|c|c|}
\hline & Isolated Rotor & Complete Helicopter \\
\hline Rotor power consumption & $130.00 \mathrm{~kW}$ & 159.30 \\
\hline
\end{tabular}

Table 8. Rotor power consumption in HLDS

$\psi<340^{\circ}$ from $0.8<r / R<1$. The descrease in pitching moment can also be extracted from Figures 25(a) and (b), showing the sectional normal force coefficient $C_{n} M a^{2}$ and sectional pitching moment coefficient $C_{m} M a^{2}$ for the radial blade stations $r / R=0.73$ and $r / R=0.87$, respectively. The sectional normal force distributions of isolated rotor and complete configuration show similar characteristics (the rotors have been trimmed for the same vertical force). However the pitching moment distributions on the retreating blade side differ significantly. The distinct peak in $C_{m} M a^{2}$ around $240^{\circ}<\psi<300^{\circ}$ is clearly visible for the $0.73 \%$ blade station and becomes even more apparent for the $87 \%$ blade station. This drop in the pitching moment distribution can be considered as an indication for Dynamic Stall, caused by a Dynamic Stall vortex shed from the leading edge blade region and passing over the airfoil.

This finding is further underlined by Figure 26. Figure 26(a) shows the surface streamline pattern on the upper blade surface for two azimuthal locations around $\psi=270^{\circ}$. One can easily identify a complex flow separation topology. The repeated change in the chordwise flow direction seen towards the inboard side of the picture is a clear indication of a Dynamic Stall vortex passing over the blade. The presence of the Dynamic Stall vortex can be verified by Figure 26(b) showing a radial cut plane through the blade mesh at $r / R=0.86, \psi=270^{\circ}$.

Finally the question arises why Dynamic Stall is only present for the complete helicopter configuration and not for the isolated rotor computation although both computations have been trimmed towards an identical trim objective. The authors assume that the rotor loading of the isolated rotor is just below the Dynamic Stall limit. The consideration of the fuselage and the tail rotor lead to changes in the rotor downwash and induced velocity distribution which finally push the rotor loading on the retreating blade side above the stall limit. This assumption holds despite the fact that the effective pitch angle on the retreating blade side is comparable for both configurations $\left(\theta=17.841^{\circ}+5.662^{\circ}\right.$ for the isolated rotor, $\theta=18.136^{\circ}+5.343^{\circ}$ for the complete configuration). As described earlier the rotor of the complete configuration features a higher longitudinal flapping amplitude which will lead to a higher downward angular flapping velocity on the retreating blade side. According to $v_{F l a p}=\dot{\beta} \cdot r$ this leads to a higher downward velocity of the blade with increasing blade radius, related to an increase of the effective sectional angle of attack. This might even compensate for the stronger nose-down blade torsion observed in Figure 20(b).

\section{Conclusions}

We have presented a weak coupling method for the main rotor between Computational Fluid Dynamics and Computational Structural Dynamics. The method has been applied to the rotor trim of a full helicopter configuration in three different flight conditions: A low-speed flight, a high-speed flight and a highly loaded rotor flight case. It has turned out that the weak coupling method is well suited for the coupled and trimmed rotor simulation of complete helicopter configurations. The method revealed very good convergence properties for all three flight cases. Small deviations of the trimmed mean CFD loads from the prescribed trim objective were observed which are related to fact that the load exchange between fluid and structure is not strictly conservative.

With respect to application we have shown that the method is able to capture the differences in the trimmed rotor solution between an isolated rotor computation and the trimmed rotor of the full helicopter configuration. Differences in both blade dynamics and aerodynamics turned out to be only minor for the low-speed flight case. For the high-speed flight case more pronounced differences in the blade torsional response and the aerodynamic disc loading were found. However, for both cases differences were found to be sufficiently small not to lead to a significant change in rotor power consumption.

Although the differences between the isolated rotor and the complete helicopter configuration in both control angles and blade dynamic response turned out to be rather small, a large difference in rotor power consumption of more than $20 \%$ was observed. The difference in rotor power consumption was found to be caused by the occurence of Dynamic Stall on the retreating blade side of the complete helicopter configuration. For the isolated rotor no Dynamic Stall was detected. A straightforward explanation of the mechanism leading to the occurance of Dynamic Stall in case of the complete helicopter is difficult to provide. The authors assume that the isolated rotor operates just below the Dynamic Stall limit. The 


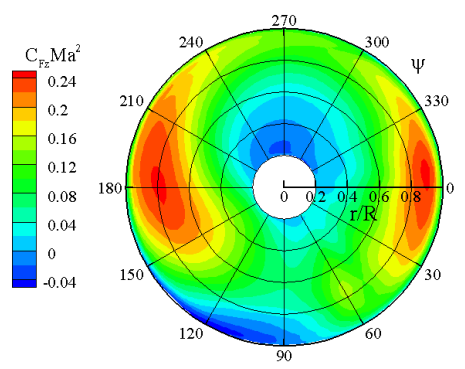

(a) Isolated Rotor

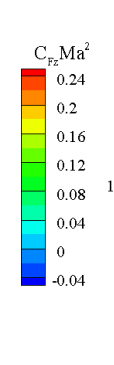

(b) Full Configuration

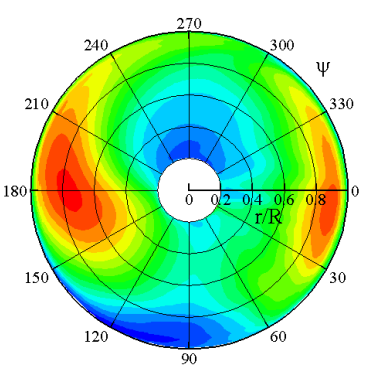

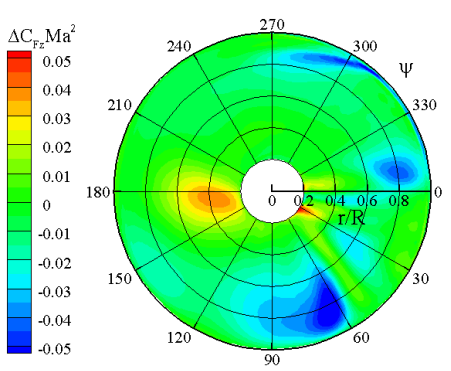

(c) Delta between Full Configuration and Isolated Rotor

Figure 22. HLDS thrust distribution on rotor disk

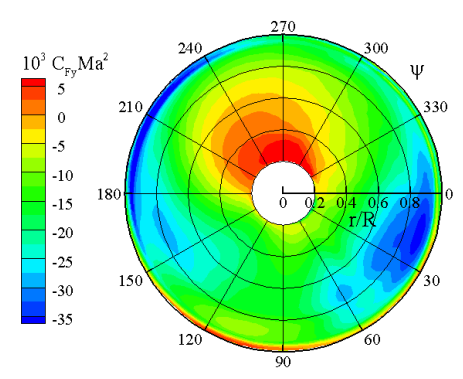

(a) Isolated Rotor
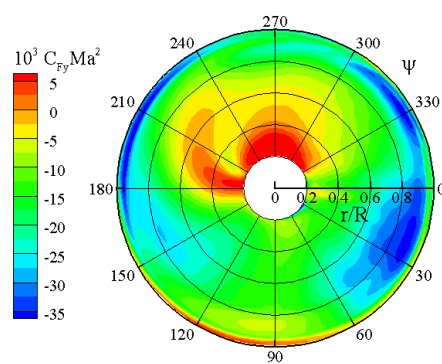

(b) Full Configuration

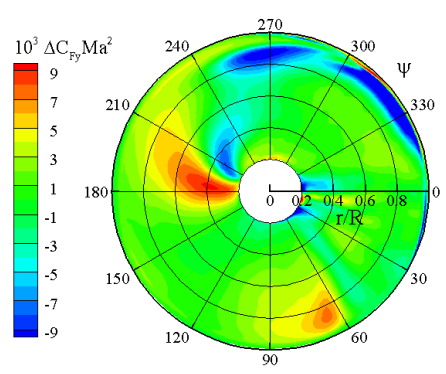

(c) Delta between Full Configuration and Isolated Rotor

Figure 23. HLDS drag distribution on rotor disk

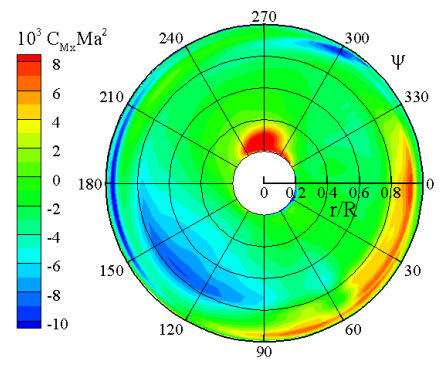

(a) Isolated Rotor

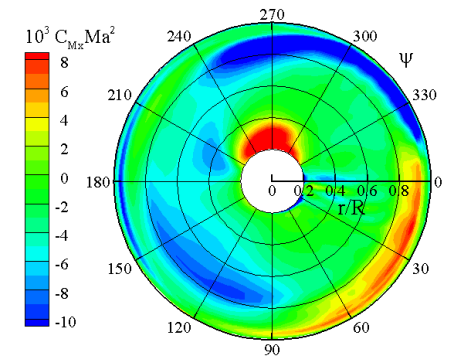

(b) Full Configuration

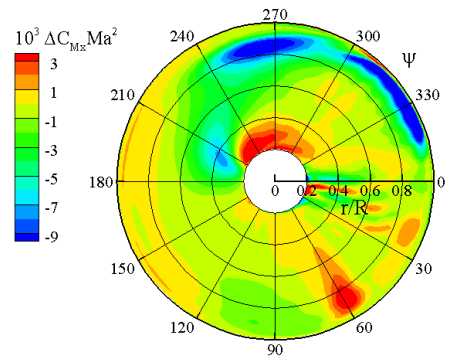

(c) Delta between Full Configuration and Isolated Rotor

Figure 24. HLDS pitching moment distribution on rotor disk

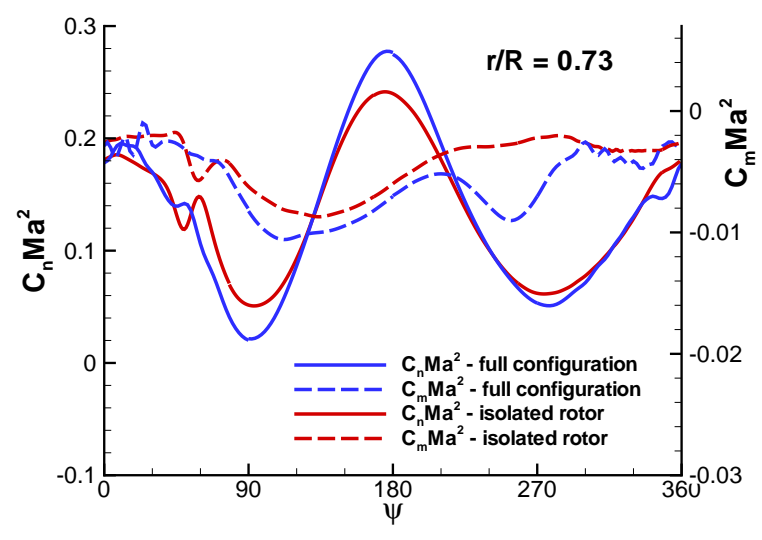

(a) $r / R=0.52$

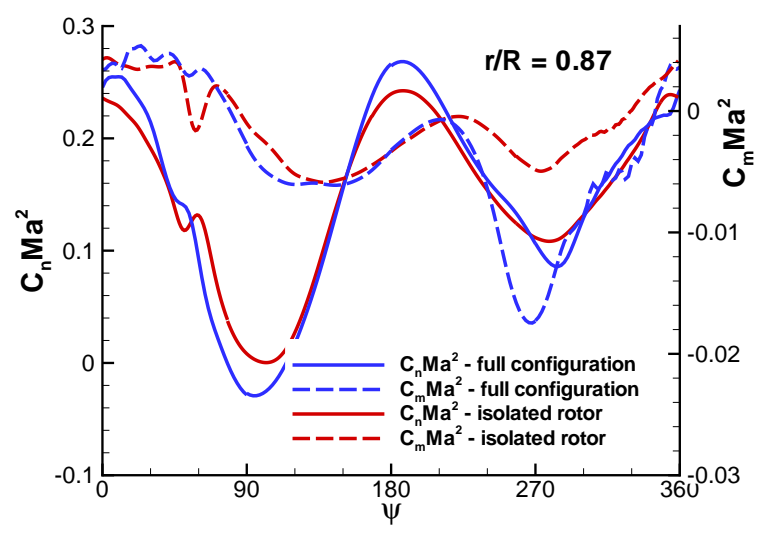

(b) $r / R=0.87$

Figure 25. Comparison of $C_{n} M a^{2}$ and $C_{m} M a^{2}$ at selected radial locations, HLDS 


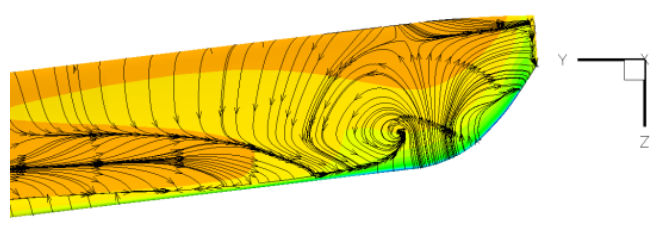

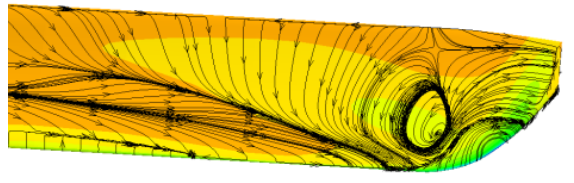

(a) Streamlines on blade surface

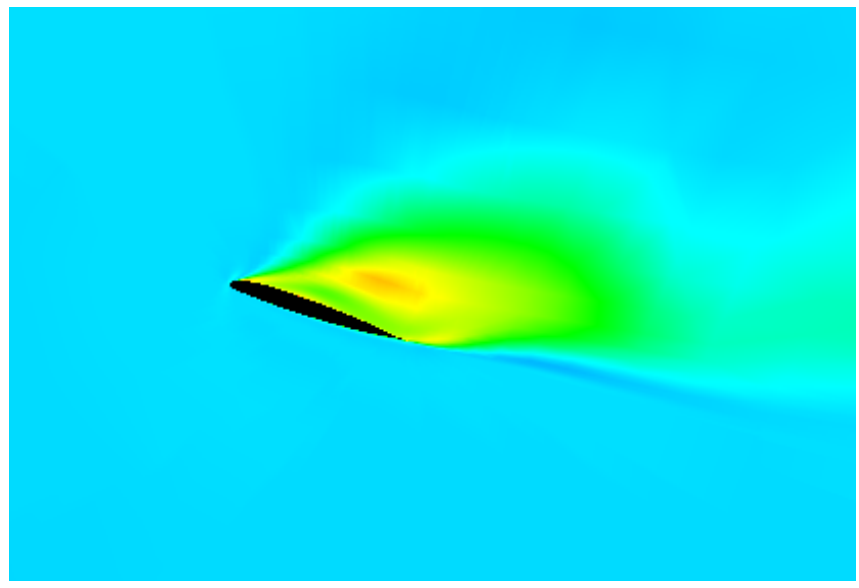

(b) Entropy contour in spanwise cut, $r / R=0.86$

Figure 26. Dynamic Stall vortex on retreating blade side, HLDS

changes in rotor downwash distribution and an increased longitudinal blade flapping motion evoked by the presence of the fuselage seem to be sufficient to push the rotor above the Dynamic Stall limit.

The present paper was intended as a numerical study. Nevertheless, as soon as the GOAHEAD wind tunnel campaign will be completed, we are going to compare our numerical simulation results of the full helicopter configuration cases to the experimental data. This will allow for a further assessment of the present findings.

\section{Acknowledgements}

The authors would like to thank the GOAHEAD consortium (DLR, ONERA, ECD, EC-SAS, AGUSTA, Westland Helicopters, CIRA, FORTH Foundation for Research and Technology, NLR, University of Glasgow, Cranfield University, Politecnico di Milano, Universität Stuttgart, Aktiv Sensor GmbH) for the fruitful cooperation and the European Union for the funding of the present work within the Specific Targeted Research Project GOAHEAD (GROWTH Contract Number AST4-CT-2005-516074).

\section{References}

\footnotetext{
${ }^{1}$ Altmikus, A., Wagner, S., Beaumier, P., Servera, G.: A Comparison: Weak versus Strong Modular Coupling for Trimmed Aeroelastic Rotor Simulations. American Helicopter Society 58th Annual Forum, Montreal, Canada, June 2002.

${ }^{2}$ Altmikus, A., Wagner, S.: On the Timewise Accuracy of Staggered Aeroelastic Simulations of Rotary Wings. AHS Aerodynamics, Acoustics and Test and Evaluations Technical Specialist Meeting, San Francisco, CA, 2002.

${ }^{3}$ Altmikus, A.: Nichtlineare Simulation der Strömungs-Struktur-Wechselwirkung am Hubschrauberrotor. Dissertation, Institut für Aerodynamik und Gasdynamik, Universität Stuttgart, Fortschritt-Berichte VDI Reihe 7, Nr. 466, ISBN 3-18-346607-4, VDI-Verlag, Düsseldorf, 2004.

${ }^{4}$ Altmikus, A., Knutzen, B.: Trimmed Forward Flight Simulation with CFD featuring Elastic Rotor Blades with and without Active Control. American Helicopter Society 63rd Annual Forum, Virginia Beach, VA, May 2007. San Francisco, CA, 2002.

${ }^{5}$ Benoit, B., Dequin, A-M., Kampa, K., Grünhagen, W.v., Basset, P-M., Gimonet, B.: Host: A General Helicopter Simulation Tool for Germany and France. American Helicopter Society, 56th Annual Forum, Virginia Beach, Virginia, May 2000.

${ }^{6}$ Boelens, O.J. ET Al.: The Blind Test Activity of the GOAHEAD Project. 33rd European Rotorcraft Forum, Kazan, Russia, September 2007.

${ }^{7}$ Boniface, J.C., Pahlke, K.: Calculations of Multibladed Rotors in Forward Flight Using 3D Euler Methods of DLR and ONERA. 22nd European Rotorcraft Forum, Brighton, England, September 1996.

${ }^{8}$ Dietz, M., Altmikus, A., Krämer, E., Wagner, S.: Weak Coupling for Active Advanced Rotors. 31st European Rotorcraft Forum, Florence, Italy, September 2005.

${ }^{9}$ Dietz, M., Krämer, E., Wagner, S.: Tip Vortex Conservation on a Main Rotor in Slow Descent Flight Using Vortex-Adapted Chimera Grids. AIAA 24th Applied Aerodynamics Conference, San Francisco, CA, June 2006.

${ }^{10}$ Dietz, M., Kessler, M., Krämer, E., Wagner, S.: Tip Vortex Conservation on a Helicopter Main Rotor Using Vortex-Adapted Chimera Grids. AIAA-Journal, Vol. 45, No. 8, August 2007, pp. 2062-2074.

${ }^{11}$ Dietz, M., Altmikus, A., Krämer, E., Wagner, S.: Active Rotor Performance Investigations Using CFD/CSD Weak Coupling. 33rd European Rotorcraft Forum, Kazan, Russia, September 2007.
} 
${ }^{12}$ Hierholz, K.-H.,WAGner, S.: Simulation of Fluid-Structure Interaction at the Helicopter Rotor. 21st ICAS Congress, Melbourne, Australia, September 1998.

${ }^{13}$ Hierholz, K.-H.: $\quad$ Ein numerisches Verfahren zur Simulation der Strömungs-Struktur-Interaktion am Hubschrauberrotor. Dissertation, Institut für Aerodynamik und Gasdynamik, Universität Stuttgart, Fortschritt-Berichte VDI Reihe 7, Nr. 375, ISBN 3-18-337507-9, VDI-Verlag, Düsseldorf, 1999.

${ }^{14}$ Jameson, A., Sсhmidt, W., Turkel, E.: Numerical Solutions of the Euler Equations by Finite Volume Methods Using Runge-Kutta Time-Stepping Schemes. AIAA-Paper 81-1259, 1981.

${ }^{15}$ Jameson, A.: Time Dependent Calculation Using Multigrid, With Applications to Unsteady Flows Past Airfoils and Wings. AIAA-Paper 91-1596, 1991.

${ }^{16}$ JeOng, J., Hussain, F.: On the Indentification of a Vortex. Journal of Fluid Mechanics, Vol. 285, pp. 69-94, 1995.

${ }^{17}$ Khier, W., Schwarz, T.: Application of Cartesian Background Grid in Combination with Chimera Method to Predict Aerodynamics of Helicopter Fuselage. 29th European Rotorcraft Forum, Friedrichshafen, Germany, September 2003.

${ }^{18}$ Khier, W., Schwarz, T., Raddatz, J.: Time-Accurate Simulation of the Flow Around the Complete Bo105 Wind Tunnel Model. 31st European Rotorcraft Forum, Florence, Italy, September 2005.

${ }^{19}$ Khier, W., Dietz, M., Schwarz, T., Wagner, S.: Trimmed CFD Simulation of a Complete Helicopter Configuration. 33rd European Rotorcraft Forum, Kazan, Russia, September 2007.

${ }^{20}$ Krämer, E., Hertel, J., Wagner, S.: Computation of Subsonic and Transonic Helicopter Rotor Flow Using Euler Equations. 13th European Rotorcraft Forum, Arles, France, September 1987.

${ }^{21}$ Kroll, N.: Computation of the Flow Fields of Propellers and Hovering Rotors Using Euler Equations. 12th European Rotorcraft Forum, Garmisch-Partenkirchen, Germany, September 1986.

${ }^{22}$ Kroll, N., Eisfeld, B., Bleecke, H.M.: The Navier-Stokes Code FLOWer. Volume 71 of Notes on Numerical Fluid Mechanics, pp. 58-71, Vieweg, Braunschweig, 1999.

${ }^{23}$ Meakin, R.L.: The Chimera Method of Simulation for Unsteady Three-Dimensional Viscous Flow. Computational Fluid Dynamics Review, Hafez, M. and Oshima, K. (Eds.), John Wiley \& Sons, pp. 70-86, 1995.

${ }^{24}$ PAhlKe, K., van DeR Wall, B.: Chimera Simulations of Multibladed Rotors in High-Speed Forward Flight with Weak Fluid-Structure-Coupling. Aerospace Science and Technologie, Vol. 9, No. 5, pp. 379-389, July 2005.

${ }^{25}$ PahlKe, K.: The GOAHEAD Project. 33rd European Rotorcraft Forum, Kazan, Russia, September 2007.

${ }^{26}$ Schwarz, T.: The Overlapping Grid Technique for the Time-Accurate Simulation of Rotorcraft Flows. 31st European Rotorcraft Forum, Florence, Italy, September 2005.

${ }^{27}$ Servera, G., Beaumier, P., Costes, M.: A Weak Coupling Method Between the Dynamics Code HOST and the 3D Unsteady Euler Code WAVES. 26th European Rotorcraft Forum, The Hague, The Netherlands, September 2000.

${ }^{28}$ Stangl, R., Wagner, S.: Calculation of the Steady Rotor Flow Using an Overlapping Embedded Grid Technique. 18th European Rotorcraft Forum, Avignon, France, September 1992.

${ }^{29}$ Steger, J., Dougherty, N., Benek, J.: A Chimera Grid Scheme. Advances in Grid Generation, Ghia, K.N. and Ghia, U. (Eds.), ASME FED, Vol. 5, pp. 59-69, New York, 1983.

${ }^{30}$ Wagner, S.: On the Numerical Prediction of Rotor Wakes Using Linear and Non-Linear Methods. AIAA 38th Aerospace Sciences Meeting \& Exhibit, AIAA-2000-0111, Reno, NV, January 2000.

${ }^{31}$ Yu, Y.H., Tung, C., van der Wall, B., Pausder, H., Burley, C., Brooks, T., Beaumier, P., Delrieux, Y., Mercker, E., Pengel,K.: The HART-II Test: Rotor Wakes and Aeroacoustics with Higher-Harmonic Pitch Control (HHC) Inputs - The Joint German/French/Dutch/US Project. American Helicopter Society, 58th Annual Forum, Montreal, Canada, June 2002. 